\title{
Existe-t-il une « Ancienne Loi des Bretons d'Armorique »? Identités ethniques et tradition manuscrite au haut Moyen Âge
}

Is there an 'ancient law of the Bretons of Armorica'? Ethnic identities and manuscript tradition in the early Middle Ages

\section{Magali Coumert}

\section{OpenEdition \\ Journals}

Édition électronique

URL : https://journals.openedition.org//bl/1162

DOI : $10.4000 / \mathrm{lbl} .1162$

ISSN : 2727-9383

Éditeur

Université de Bretagne Occidentale - UBO

\section{Édition imprimée}

Date de publication : 1 mars 2014

Pagination : 227-264

ISBN : 979-10-92331-07-3

ISSN : $1270-2412$

\section{Référence électronique}

Magali Coumert, «Existe-t-il une «Ancienne Loi des Bretons d'Armorique » ? Identités ethniques et tradition manuscrite au haut Moyen Âge », La Bretagne Linguistique [En ligne], 18 | 2014, mis en ligne le 01 mai 2021, consulté le 21 mai 2021. URL : http://journals.openedition.org//bl/1162 ; DOI : https:// doi.org/10.4000/lbl.1162

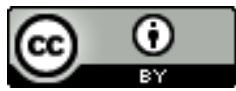

La Bretagne Linguistique est mise à disposition selon les termes de la Licence Creative Commons Attribution 4.0 International. 
Magali COUMERT*

\section{Existe-t-il une «Ancienne Loi des Bretons d'Armorique »? Identités ethniques et tradition manuscrite au haut Moyen Âge}

$\mathrm{U}_{\mathrm{u}}$

ne série de dispositions juridiques publiée par L. Bieler sous le titre de canones Wallici $^{1}$ a été considérée par L. Fleuriot comme «Un fragment en latin de très anciennes lois bretonnes armoricaines $\mathrm{du} \mathrm{VI} \mathrm{V}^{\mathrm{e}}$ siècle $»^{2}$. Plus récemment, S. Kernéis l'appelle «L'ancienne loi des Bretons d'Armorique ${ }^{3}{ }^{3}$ et considère qu'elle a été composée dans l'empire romain pour une communauté militaire du IVe siècle ${ }^{4}$. Pourtant les manuscrits donnent le plus souvent comme titre de ce texte anonyme : Extraits des livres des Romains et des Francs, et ne

* Maître de conférences d'histoire médiévale, CRBC (EA 4451-UMS 3554), $\mathrm{UBO} / \mathrm{ueb}$.

1. Ludwig Bieler, The irish penitentials, Dublin, Scriptores latini Hiberniae V, 1re edition 1963, 2e éd. 1975, p. 136-159, désormais BIELER, 1963.

2. Léon Fleuriot : c'est le titre de son article paru dans les Annales de Bretagne, t. 78, 1971, p. 601-660, désormais FLEURIOT, 1971.

3. Soizig Kerneis, «L'ancienne loi des Bretons d'Armorique. Contributions à l'étude du droit vulgaire», Revue historique de droit français et étranger 73 (2), 1995, p. 175-199, désormais KERNEIS, 1995.

4. Sur l'origine et l'inadéquation de cette hypothèse, voir M. COUMERT, «Des lois bretonnes du Haut Moyen Âge ? Les Extraits des livres des Romains et des Francs», à paraitre dans les Mélanges à la mémoire de B. Merdrignac, dans la collection Britannia Monastica. 
mentionnent jamais ni le terme de Bretagne ni celui de breton à son propos.

À travers cet exemple particulier pris dans mon domaine de spécialité, le haut Moyen Âge occidental, je voudrais discuter des critères qui peuvent être retenus pour associer un écrit, en l'occurrence un texte à caractère juridique, et une communauté ethnique, ici les Bretons d'Armorique. En d'autres termes, pour quelles raisons affirmer l' "Armoricité» d'un texte médiéval sans auteur connu? L'argumentation des historiens s'appuie sur l'étude des six manuscrits qui comportent ce texte particulier, ainsi que des gloses en langue vernaculaire et des noms propres. L'interprétation de ces éléments a pourtant beaucoup évolué ces dernières années, tant il apparait que les préoccupations nationalistes des $\mathrm{XIX}^{\mathrm{e}}$ et $\mathrm{XX}^{\mathrm{e}}$ siècles ont été plaquées sur les identités ethniques et les langues du haut Moyen Âge. Un nouvel examen des différents éléments mis en avant peut aboutir à remettre en cause ce qui apparaissait démontré il y a seulement une trentaine d'années. Nous rappellerons tout d'abord le cadre historiographique dans lequel fut étudiée la question, avant de chercher le sens de la copie des Extraits des livres des Romains et des Francs au sein de chaque manuscrit qui les comporte.

\section{Les attributions à des communautés ethniques}

En 1963, Ludwig Bieler éditait les pénitentiels irlandais dans la collection des Scriptores latini Hiberniae ${ }^{5}$. À coté des pénitentiels se trouve une série de dispositions juridiques appelée Canones Wallici ou «welsh canons», un titre hérité ${ }^{6}$ qui semble peu satisfaisant à l'éditeur, qui ne le donne qu'entre guillemets. Il ne correspond pas aux six manuscrits, copiés entre le $\mathrm{IX}^{\mathrm{e}}$ et le $\mathrm{XI}^{\mathrm{e}}$ siècle, qui portent quatre fois comme titre «Ici commencent les extraits des livres des Romains et des Francs»?

5. Voir BIELER 1963.

6. Le titre, erroné et absent des manuscrits, vient du premier éditeur, Hermann (F. W. H.) WASSERSCHLEBen, Die Bussordungen der abendländischen Kirche, Halle, 1851, p. 124-136.

7. Manuscrit $A$ : Orléans, Bibliothèque municipale, 221, p. 206-212.

Manuscrit B : Paris, Bibliothèque Nationale de France (BNF), latin 3182, p. 160164. 
Ces «Extraits» se présentent comme une liste de 63 à 67 chapitres qui indiquent des peines pour des homicides, des blessures, des relations sexuelles illicites, mais aussi des vols d'animaux. Ces peines sont exprimées sous des formes différentes : esclaves à payer, jugement, remise aux mains des parents de la victime, abandon de toute poursuite contre l'assassin, ou amende exprimée en livres ou sous d'argent. Aucune autorité publique supérieure n'est évoquée, mais l'organisation sociale apparaît à travers un tribunal ${ }^{8}$, un juge ${ }^{9}$ et des anciens ${ }^{10}$, un fisc ${ }^{11}$ et un tributaire ${ }^{12}$, parfois une cité ${ }^{13}$.

Les dispositions envisagent une société chrétienne, avec une église ${ }^{14}$, un clerc ${ }^{15}$, la confession à un prêtre ${ }^{16}$, un appel à Dieu

Manuscrit H : Oxford, Bodleain Library, Hatton 42 (section A), fol. 130r-132v.

Manuscrit O : Londres, British Library, Cotton Otho E. XIII, fol. 139r-141v.

Manuscrit X : Cambridge, Corpus Christi College, 265, p. 100-104.

Manuscrit P : Paris, BNF, latin 12021, fol. 135r-138r.

Pour le titre, les manuscrits ABXH donnent : Incipiunt excerpta de libris (libri X) Romanorum (Romanis A) et Francorum. Les deux autres manuscrits varient de façon importante : ms.O : sinod( ?) rom( ?) incipi(unt) pauca Colomella ; ms. P : Incipit iudicium culparum.

8. A 3 .

9. A 30, A 36, A 37, P 41 .

10. A 36 .

11. A 45, P 49 .

12. A 30 : tributarius. L. Bieler traduit comme le percepteur du tribut, mais le latin indique à l'inverse un tributaire, ainsi que le remarque L. FLEURIOT, «Les très anciennes lois bretonnes. Leur date. Leur texte», dans Landévennec et le monachisme breton dans le haut Moyen Âge. Actes du colloque du $15^{e}$ centenaire de l'abbaye de Landévennec, 25-26-27 avril 1985, Landévennec, 1986, p. 65-84 (désormais FLEURIOT 1986), ici p. 81. L'usage de l'adjectif tributarius, $a$, um, bien attesté dès l'époque classique pour tributaire, sous une forme substantivée se trouve par exemple dans la loi salique (Pactus legis salicae, 41, 10, K.A. Eckhardt éd., Monumenta Germaniae Historica, Leges nationum germanicarum 4, 1, Hanovre, 1962). Dans S. KERNÉIS 1995, p. 175-199, l'auteur relève p. 192 l'hapax tributatorius du manuscrit $\mathrm{A}$, pour lequel elle décrit une fonction juridique particulière, sans justifier son refus de la tradition manuscrite majoritaire. Je ne présente pas ici la réfutation des hypothèses que l'auteur avance dans cet article, que j'ai développée dans l'article à paraitre pour les Mélanges B. Merdrignac.

13. A 63, qui n'est présent que dans les manuscrits A et B.

14. A 4, P 3.

15. A $43, \mathrm{P} 44-45$.

16. A 37. 
comme témoin ${ }^{17}$, le jugement d'un clerc par des évêques ${ }^{18}$, ainsi qu'une condamnation par l'exclusion de l' «Église de Dieu et de la table des chrétiens ${ }^{19}{ }^{1}$. Le texte est écrit en latin, et évoque, suivant les manuscrits, des chevaux achetés à des Calpei ${ }^{20}$, des Calfaices ${ }^{21}$, des Galli $^{22}$ ou des Saxones ${ }^{23}$, ce qui ne permet pas de localiser le lieu de sa composition dans une communauté chrétienne du nord-ouest de l'Europe, dans les îles britanniques ou sur le continent.

L'éditeur distingue deux recensions différentes, $\mathrm{A}$ et $\mathrm{P}^{24}$, d'un texte qu'il interprète comme une collection de lois pour une communauté rurale indéfinie ${ }^{25}$, car seules les trois dernières dispositions, qui lui semblent avoir été ajoutées, ont un caractère pénitentiel ${ }^{26}$. Il s'éloigne donc de l'opinion du premier éditeur, H. Wasserschleben, en soulignant le caractère laïc des dispositions juridiques, mais reprend son interprétation en considérant que leur base était essentiellement galloise ${ }^{27}$.

\section{Le classement ethnique des lois du haut Moyen Âge}

Avant de détailler les raisons de cette attribution à la communauté galloise, ou sa réfutation au profit de la communauté bretonne continentale, il faut souligner l'apparente nécessité, pour les éditeurs successifs, d'attribuer cette liste de peines et d'amendes à un groupe ethnique particulier. Or le texte, contrairement à un grand nombre de législations du haut Moyen Âge, comme la Loi salique, l'édit

17. A $44, \mathrm{P} 48$.

18. A 37.

19. A 59 et A 61 .

20. A 20 suivant le manuscrit $H$.

21. P 29. Fleuriot 1971, p. 616-617 propose d'y lire le nom du peuple des Taïfales.

22. A 20 suivant les manuscrits A, B, H et les gloses mineures de $\mathrm{H}$.

23. A 20 et P 29, dans tous les manuscrits.

24. Contenue exclusivement dans le manuscrit $P$.

25. L. Bieler qualifie le texte de «old village code» dans L. BIELER, «Towards an interpretation of the so-called 'Canones Wallici'», dans S.J.J.A. Watt, J. B. Morrall et F.X. Martin dir., Medieval Studies Presented to Aubrey Gwynn, Dublin, 1961, p. 387-392, ici p. 389, note 11.

26. Version A, dispositions 59-61. BIELER, 1963, p. 7 : «They are not penitential but a collection of laws of some rural community.»

27. L. BIELER, op. cit. à la note 25 . 
de Rothari ou les codes des rois anglo-saxons ${ }^{28}$, n'évoque jamais l'appartenance ethnique, aussi bien pour les parties que pour le juge. Le coupable est désigné le plus souvent par le pronom indéfini quis. Il n'est que rarement distingué entre son statut de libre ou d'esclave ${ }^{29}$, comme de clerc ou de laïc ${ }^{30}$. Une minorité, avant 12 ou 15 ans, lui est parfois reconnue ${ }^{31}$. Mais jamais il n'est prévu de variation de la peine suivant une appartenance ethnique. Le titre, qui évoque les «livres des Romains et des Francs», ne distingue pas ensuite ses emprunts par leur origine. Les identités ethniques ne sont pas prises comme base d'un jugement différencié.

Pourtant, les éditeurs puis les commentateurs de ces Extraits des livres des Romains et des Francs se sont efforcés d'en circonscrire l'application éventuelle à une communauté ethnique particulière. Cette attitude nous renvoie au fait que le développement de l'histoire, en tant que discipline scientifique, au cours des $\mathrm{XIX}^{\mathrm{e}}$ et $\mathrm{XX}^{\mathrm{e}}$ siècles, a accompagné celui des nationalismes européens. Ainsi, la publication des différents codes de lois du haut Moyen Âge pour les peuples de langue germanique, supposés les ancêtres des Allemands, est au cœur du projet nationaliste d'édition des sources du Moyen Âge allemand porté par la «Gesellschaft für ältere deutsche Geschichtskunde» fondée en $1819^{32}$. Elle a publié la prestigieuse collection des Monumenta Germaniae Historica ( $M G H)$, qui fut très rapidement une référence pour la qualité de ses éditions de textes médiévaux.

Dès le départ, les Monumenta s'intéressèrent aux recueils juridiques du haut Moyen Âge, et lancèrent notamment à partir de 1888 une nouvelle série sur les lois des nations germaniques ${ }^{33}$, soustitrée «Sammlung der Volksrechte» ${ }^{34}$. Cette expression peut très

28. Sur ces questions, voir M. COUMERT, «Lois barbares et identité ethnique», dans le volume à paraitre sous la direction de $\mathrm{M}$. Candido et $\mathrm{S}$. Joye, Les lois barbares du haut Moyen Âge.

29. Cas d'un coupable libre : A 7 (P 6), A 27 (P 21 et 22), ou esclave A 5, (P 4), A 33, (P 38), ou maitre A 6 (P 5).

30. A 37, (P 40 et 41); A 43 (P 44), ; 56 (P 66); A 57 (P LXV).

31. A 26 et $\mathrm{P} 18$.

32. Societas aperiendis fontibus rerum Germanicarum medii aevi.

33. Leges nationum Germanicarum.

34. Par exemple, MGH, Leges nationum Germanicarum, Tome V, 1, Leges Alamannorum, K. Lehmann éd., Hanovre, 1888, page de garde. 
imparfaitement être traduite par «collection des lois nationales», car dans l'expression «Volksrechte», le peuple, «Volk», envisagé n'est pas le résultat d'un pacte d'adhésion républicain, que sous-entend le terme français de nation, non plus qu'une classe sociale. Le «Volk» germanique ici envisagé reprend une conception romantique : c'est un groupe d'ascendance commune, distinct culturellement et génétiquement des autres depuis les origines, qui fonde la légitimité de ses lois sur leur transmission coutumière. Les collaborateurs des Monumenta se concentrèrent exclusivement sur les peuples qu'ils assimilaient à leurs ancêtres, dès lors qu'ils pouvaient leur attribuer une langue de type germanique, ou un rapport avec le territoire appelé, à leurs yeux, à former une Allemagne unifiée. Nous trouvons ainsi édités dans des volumes distincts les lois des Goths, des Francs, des Bavarois, des Alamans...

En dehors de la collection pionnière des Monumenta, qui faisait autorité en terme de démarche d'établissement du texte, les textes des lois barbares ont été édités suivant le même principe, en se concentrant sur une seule identité ethnique. On trouve ainsi rassemblées les lois des Anglo-Saxons, des Lombards ${ }^{35}$... Le classement par identité ethnique séparée et les regroupements linguistiques, suivant la distinction celtique ou germanique, ont ainsi sous-entendu tout le travail des éditeurs des lois des souverains occidentaux, alors même que de telles conceptions paraissent anachroniques pour le haut Moyen Âge : les regroupements comme celtique ou germanique n'existent pas aux yeux des érudits ${ }^{36}$ et la langue apparait très rarement comme un argument de pouvoir alors que les textes impliquant une autorité publique sont presque toujours écrits en latin.

En outre, les éditions des différents volumes furent séparées des codes de droit romain et organisées par identité ethnique, suivant la conviction que chacun se référait au haut Moyen Âge au droit spécifique de son ethnie et que celui-ci était défini par des traditions orales. En effet, suivant l'historiographie alors dominante, la person-

35. Par exemple, F. Liebermann, Die Gesetze der Angelsachsen, Berlin, 1903, ou plus récemment, Cl. AzZARA et S. GASPARri, Le leggi die Longobardi. Storia, memoria e diritto di un popolo germanico, Milan, 1992.

36. Voir M. Coumert, «Hraban Maur et les Germains», Ph. Depreux, S. Lebecq, M. J.-L. Perrin et O. Szerwiniack dir., Raban Maur et son temps, Turnhout, 2010, collection Haut Moyen Âge 9, p. 137-153. 
nalité des lois aurait été un héritage des grandes migrations ${ }^{37}$. Cette conception d'un peuple et de ses traditions propres explique l'édition en volumes séparés du droit attribué à chaque peuple, en fabriquant une coupure artificielle avec l'Antiquité. Ainsi, le Bréviaire d'Alaric, un recueil commenté de droit romain publié par le roi des Wisigoths Alaric II en 506, n'est pas publié dans le volume rassemblant les leges Wisigothorum. Il s'appliquait pourtant aux Romains comme aux Goths et fut repris par Clovis pour son royaume ${ }^{38}$.

Notre regard sur ces textes juridiques a été entièrement biaisé par ces désignation par groupe ethnique, et une édition séparée des différentes lois que la tradition manuscrite contredit totalement : une écrasante majorité des manuscrits conservés rassemblent en effet les textes attribués à différentes identités ethniques. Ainsi, sur les 49 manuscrits utilisés par Ernest de Schwind pour éditer la «loi des Bavarois » ${ }^{39}, 18$, soit plus d'un tiers, comportent aussi la loi salique. Les éditions ont donc créé des barrières autour de chaque code de lois, alors même que si la plupart se réfèrent bien à des identités ethniques particulières, la tradition écrite démontre qu'ils n'ont jamais suscité un intérêt isolé de l'héritage juridique antique et des lois des groupes voisins.

\section{Un code de lois gallois ou breton?}

Les Extraits des livres des Romains et des Francs se trouvent dans des manuscrits qui, par leurs gloses, ont tout d'abord suscité l'intérêt des linguistes spécialistes des langues celtiques, comme Whitley Stokes à la fin du XIX ${ }^{\mathrm{e}}$ siècle $^{40}$. Leur entreprise de reconstruction des langues celtiques médiévales, à partir des manuscrits

37. Sur l'évolution de cette conviction, voir Patrick WoRMALD, «The leges barbarorum : law and ethnicity in the post-roman west», p. 21- 53, dans H.W. Goetz, J. Jarnut et W. Pohl dir., Regna und gentes. The Relationship between Late Antique and Early Medieval Peoples and Kingdoms in the Transformation of the Roman World, Leyde/Boston, Brill, 2003, coll. The Transformation of the Roman World, vol. 13) et le volume annoncé à la note 28 .

38. Voir de façon générale : M. Rouche et B. DumézIL (dir.), Le Bréviaire d'Alaric. Aux origines du Code civil, Paris, PUPS, 2008.

39. MGH, Leges nationum germanicarum, V, 2, Hanovre, 1926.

40. Sur celui-ci, voir Henri D’Arbois de Jubainville, «Whitley Stokes, associé étranger de l'Académie des Inscriptions et Belles-Lettres », Journal des savants. 7e année, Juin 1909, p. 284-286. 
conservés sur le continent, a accompagné la recherche des textes qui purent être composés dans les milieux utilisant ces langues, pour reconstituer une culture celtique médiévale spécifique.

Dans le cadre général tracé par les éditions des Monumenta, en accord avec les travaux de l'école juridique allemande antérieure à la Seconde Guerre mondiale, où la conception romantique du peuple s'alliait avec l'idée de coutumes spécifiques à chaque peuple, transmises oralement avant leur mise par écrit, les groupes ethniques auxquels était associée une langue celtique paraissaient bien dépourvus de droit ancestral pour les premiers siècles du Moyen Âge. Les dispositions juridiques des Extraits des Livres des Romains et des Francs semblaient par bien des aspects pouvoir combler ce qui apparaissait comme un vide. Ainsi, pour L. Fleuriot, «ces lois laïques très anciennes existaient depuis des siècles sous une forme orale dans les pays celtiques et il ne fait pas de doute que l'enseignement des druides portaient en partie sur ces textes ${ }^{41} \gg$. Mais en l'absence totale d'indication ethnique en dehors du titre, plusieurs peuples pouvaient en bénéficier.

Ainsi, H. Bradshaw et $\mathrm{H}$. Wasserschleben débattaient déjà, à la fin du XIX ${ }^{\mathrm{e}}$ siècle, pour attribuer ou non une origine bretonne aux manuscrits comportant les Extraits des livres des Romains et des Francs $^{42}$. En revanche, suivant L. Bieler, la base en serait essentiellement galloise ${ }^{43}$ en raison des éléments suivants :

- une partie de ces dispositions a été reprise dans les résumés latins des lois du roi gallois Hywel Dda du $\mathrm{X}^{\mathrm{e}}$ siècle. Néanmoins, le plus ancien manuscrit date du XIII ${ }^{\mathrm{e}}$ siècle, et elles ne se trouvent pas dans le texte gallois original ;

- des amendes en nature, poids de métal ou esclaves, telles qu'elles apparaissent dans ce texte existent en Irlande et au pays de Galles au haut Moyen Âge ;

41. L. Fleuriot, «Les recueils bretons de Lois religieuses et civiles du très haut Moyen Âge», Études sur la Bretagne et les pays celtiques. Mélanges offerts à Yves Le Gallo, Brest, 1987, p. 113-118, ici p. 114.

42. Voir les arguments déjà échangés sur ce sujet par H. BRADSHAW et H. WASSERSCHLEBEn, Die irische Kanonensammlung, $2^{\mathrm{e}}$ édition, Leipzig, 1885, p. LXVIILXXII, étudiés par L. FLEURIOT dans l'article cité à la note précédente.

43. L. BIELER, op. cit. à la note 25 . 
- 4 chapitres ${ }^{44}$ se réfèrent à la compurgation, qui existe aussi dans les procédures légales galloises.

Néanmoins, les liens avec le continent lui paraissent soulignés par le titre de l'ensemble, ainsi que les parallèles entre les descriptions des actes délictueux dans les Extraits des livres des Romains et des Francs et la loi salique ${ }^{45}$.

L. Bieler étudie alors les liens entre les différents manuscrits et la Bretagne : 3 d'entre eux lui paraissent avoir été copiés par des Bretons, tandis que 4 sur 6 comportent à ses yeux des gloses en vieux breton. Il conclut donc qu'il s'agit d'un ancien texte gallois apporté en petite Bretagne par des réfugiés. Leurs anciennes traditions auraient alors été modifiées au contact des Francs. Les «Romains» du titre seraient alors les Britto-romains qui s'enfuirent en petite Bretagne devant les Angles et les Saxons ${ }^{46}$.

L. Fleuriot a contesté cette interprétation, en considérant que l'inclusion dans l'abrégé latin des lois de Hywel Dda était attestée de façon trop tardive pour indiquer l'origine du texte ${ }^{47}$. Il affirme au contraire :

«L'origine armoricaine du texte résulte : 1) de la tradition manuscrite ; 2) des gloses en vieux breton, à l'exclusion de toute autre langue non latine ; 3 ) des noms des scribes et des abbés mentionnés dans les manuscrits eux-mêmes ; 4) de la comparaison du texte avec les autres lois celtiques, plus particulièrement les lois galloises ${ }^{48} . »$

44. A 4 (=P 3), A 18 (= P 11), A 31 (= P 32)e t A 43 (= P 45).

45. A 5 (= P 4) avec Pactus legis salicae (K.A. Eckhardt éd. comme note 12, désormais $P L S) 35,8$;

A 8-9 (= P 7) et PLS 29,1 et 4 ;

A $10(=\mathrm{P} 8)$ et $P L S 17,6$;

A 11 (= P 9) et $P L S 17,4$;

A $12(=\mathrm{P} 51)$ et $P L S 17,8$;

A 13 (= P 36) et PLS 17,9;

A $18(=\mathrm{P} 11)$ et $P L S 34,4$;

A 33 (= P 38) et PLS 35,1.

46. Voir M. Coumert, «Le peuplement de l'Armorique», dans M. Coumert et H. Tétrel dir., Histoires des Bretagnes. Les mythes fondateurs, Brest, CRBC, 2010, p. 15-42 pour un bilan des questions soulevées par cette présentation traditionnelle.

47. Fleuriot, 1971, p. 607.

48. FLEURIOT, 1971, p. 612. 
Il reconnait lui aussi les Britanni derrière les «Romains» mentionnés dans le titre. Considérant que la loi salique a été composée à la fin du règne de Clovis, il suppose que la composition des Extraits des Livres des Romains et des Francs eut lieu entre 520 et 560, avant une période d'hostilités entre Francs et Bretons armoricains.

En examinant à son tour le dossier ${ }^{49}, \mathrm{D}$. Dumville a accepté le principe d'une origine bretonne de ces dispositions légales, tout en reconnaissant que les bases de cette hypothèse restaient fragiles ${ }^{50}$. Il a contesté la datation proposée par Fleuriot, qui repose sur l'interprétation de sources hagiographiques postérieures au $\mathrm{IX}^{\mathrm{e}}$ siècle. Le seul terminus ante quem que l'on puisse à ses yeux retenir est celui fourni par la date du plus ancien manuscrit, soit la première moitié du IX $\mathrm{IX}^{\mathrm{e}}$ siècle $^{51}$. En outre, l'analyse des relations textuelles entre les Extraits et la Collectio canonum hibernensis (versions A et $\mathrm{B}$ ) l'incline à penser que certains chapitres des premiers dérivent de ces deux versions de la collection canonique, et non de leurs sources, ce qui lui fait dater la composition des Extraits au plus tôt du VIII siècle $^{52}$.

En réponse à cette étude, L. Fleuriot a donné une traduction des Extraits des livres des Romains et des Francs et répondu qu'il distinguait les extraits tels qu'ils nous sont parvenus des lois dont ils sont issus ${ }^{53}$. Pour lui, la composition de ces lois laïques serait antérieure même aux pénitentiels irlandais, qu'elles auraient inspirés. La distinction des Romani et des Franci dans le titre soulignerait l'antiquité de la composition du document. Le premier terme désignerait les Bretons, «mais le contenu même des Excerpta est celtique et non romain ${ }^{54} \gg$.

L'argumentation paraît biaisée, dès lors qu'elle se réfère à un hypothétique état initial du texte, sous forme orale ou écrite, qui ne nous est pas parvenu. Par la suite, l'historiographie a en général re-

49. D. N. DumVille, «On the dating of the early Breton lawcodes», Études celtiques XXI, 1984, p. 207-221.

50. Ibid., p. 209 : «The Breton manuscript-tradition is not a guarantee of Breton origin»".

51. Ibid., p. 213.

52. Ibid., p. 216-220. Voir infra pour la datation de la collectio canonum hibernensis.

53. L. FleURIOT, «Les très anciennes lois bretonnes...», op. cit. note 12, p. 65-84.

54. Fleuriot, 1986, p. 68. 
tenu l'attribution des anciens «Canones Wallici» aux Bretons d'Armorique, mais pas la datation haute proposée par L Fleuriot. Ainsi, G. Le Duc considère qu'il s'agit des « anciennes lois bretonnes ${ }^{55}$ » et affirme : «Les Canones Wallici ont longtemps été considérés comme des lois galloises, jusqu'à ce qu'en 1971 le Pr. Fleuriot, dans un article qui est devenu classique, démontre leur origine armoricaine», sans pour autant reprendre la question de la datation ${ }^{56}$.

L'argumentation pour définir le caractère «breton» de ce texte repose donc principalement sur sa tradition manuscrite (arguments 1, 2 et 3 de Fleuriot en 1971), et non sur les dispositions légales du texte, qui se rapprochent bien de certaines normes insulaires, mais aussi continentales à travers les parallèles avec la loi salique et les livres évoqués par le titre. Il nous faut donc revenir sur les critères retenus pour attribuer les six manuscrits qui comportent les Extraits des livres des Romains et des Francs à une communauté ethnique particulière.

\section{La tradition manuscrite des Extraits des livres des Romains et des Francs : des manuscrits bretons?}

\section{La collectio canonum Hibernensis}

Cette enquête sur la tradition manuscrite des Extraits des livres des Romains et des Francs tournera autour de la Collectio canonum hibernensis puisque cinq manuscrits sur les six les contenant la comportent aussi, dans sa version A, B ou C. Il s'agit d'une collection de canons compilée entre 690 et 748 . Le colophon du manuscrit P l'attribue à des clercs de communautés scotes du sud de l'Irlande et du nord-ouest de l'Angleterre ${ }^{57}$. Néanmoins, une préface contenue dans

55. G. LE Duc, «Notes sur les anciennes lois bretonnes (Canones Wallici)», Bretagne et pays celtiques. Langue, histoire, civilisation. Mélanges offerts à la mémoire de Léon Fleuriot (1923-1987), Rennes, Saint-Brieux, 1992, p. 101-109.

56. De même dans G. LE Duc, «Les premiers temps de l'évêché de Quimper», Britannia Monastica 3, Landévennec, 1994, p. 85-168, ici p. 88.

57. Paris, BNF, latin 12021, fol. 139r : «jusqu'ici, Ruben de Dairinis et Cu Cuimni d'Iona», Hucusque Ruben et Cu Cuimni Iae et Daniris. Voir D. N. DumviLle, «Ireland, Brittany, and England : transmission and use of the Collectio canonum hibernensis », dans Irlande et Bretagne. Vingt siècles d'histoire, C. Laurent et H. Davis dir., Rennes, 1994, p. 85-95, et Bart JASKI, «Cú Chuimne, Ruben and the Compilation of the Collectio Canonum Hibernensis», Peritia 14, 2000, 
trois manuscrits est, quant à elle, à la première personne du singu$\operatorname{lier}^{58}$ : il y eut donc, semble-t-il, différentes étapes de composition de cette collection, comme l'indique l'existence de plusieurs versions ${ }^{59}$, dont une étape liée à l'Irlande Elle ne subsiste plus néanmoins que dans des manuscrits continentaux, dont le plus ancien remonte au milieu du VIII' siècle $^{60}$.

Cette collection a été recopiée et diffusée sur le continent dès le VIII ${ }^{\mathrm{e}}$ siècle, portée par la réforme carolingienne de l'Église ${ }^{61}$. Elle témoigne de l'intégration par étapes de textes d'origines diverses par l'ensemble de la chrétienté latine, comme l'a montré H. Mordek : elle ne permet pas de préciser l'ancrage spatial des différents manuscrits qui la contiennent ${ }^{62}$.

p. 51-69. J'ai lu David Howlett, «The Prologue to the Collectio Canonum Hibernensis», Peritia 17-18, 2003-4, p. 144-149, mais si les remarques sur le style recherché, par le rythme et les assonances, me semblent justifiées, les décomptes numériques me paraissent jouer des variantes manuscrites, pour aboutir aux chiffres souhaités. Si de tels décomptes avaient été considérés comme significatifs, pourquoi les copistes ne les auraient-ils pas respectés?

58. Cette préface est présente dans le manuscrit P (Paris, BNF, latin 12021) qui comporte la recension A de la collectio canonum hibernensis, ainsi que dans les manuscrits H (Oxford, Bodleian Library, Hatton 42) et le manuscrit de Rome, Biblioteca Vallicelliana, T. XVIII qui comportent la recension B.

59. Lunedd Mair DAvies, «Isidorian Texts and the Hibernensis», Peritia 11, 1997, p. 207-249, ici p. 212-214.

60. Rob MeEns, «The oldest manuscript witness of the Collectio canonum hibernensis », Peritia 14, 2000, p. 20-50. La liste des manuscrits et une bibliographie sont fournies par Lotte KÉRY, Canonical Collections of the Early Middle Ages (ca. 400-1140). A bibliographical Guide to the Manuscripts and Literature, Washington, 1999, p. 73-80.

61. H. MordeK, Kirchenrecht und Reform im Frankenreich. Die Collectio Vetus Gallica, die älteste systematische Kanonessammlung des fränkischen Gallien, Berlin/New York, 1975, p. 255-259 et Roger E. REYNOLDS, «Unity and Diversity in Carolingian Canon Law Collections: The Case of the Collectio Hibernensis and Its Derivatives ", Carolingian Essays. Andrew W. Mellon Lectures in Early Christian Studies, U. R. Blumenthal dir., Washington, 1983, p 98-135.

62. Helen Simpson McKeE, «Breton manuscripts of biblical and hiberno-latin texts », dans The Scriptures and Early medieval Ireland. Proceedings of the 1993 Conference of the Society for Hiberno-Latin Studies on Early Irish Exegesis and Homiletics, Th. O'Loughlin dir., Turnhout, Brepols, 1999, p. 275-290, ici p. 286288. 
Les relations entre les différentes versions de la Collectio canonum hibernensis restent à explorer ${ }^{63}$. L. Mair Davies a repéré que les citations d'Isidore de Séville qui l'émaillent prennent, dans certains cas, une forme particulière dans les manuscrits ABHOP, qui contiennent aussi les Extraits des livres des Romains et des Francs ${ }^{64}$. Comme elle tient pour acquise l'origine bretonne de ces manuscrits, elle formule l'hypothèse d'une version bretonne de la Collectio canonum hibernensis, qui serait intermédiaire entre les versions A et $\mathrm{B}^{65}$.

L'absence de réelle édition critique de la Collectio ne permet pas d'approfondir cette hypothèse ${ }^{66}$. Nous retiendrons néanmoins que ces observations viennent souligner la proximité entre les différents manuscrits qui comportent les Extraits, et leur position dans une famille textuelle distincte de la Collectio canonum hibernensis. Néanmoins, l'association de cette version de la collection canonique et de la Bretagne continentale étant ici le fruit de la réception de l'historiographie antérieure, elle doit être revue après examen de celle-ci. En quoi $\mathrm{ABHO}$ et $\mathrm{P}$ peuvent-ils être considérés comme des manuscrits bretons?

Il nous faut donc détailler les six manuscrits comportant les Extraits des Livres des Romains et des Francs, tous copiés à l'époque carolingienne, pour tenter de préciser leur origine. Cinq sur six présentent des noms de copistes et des gloses en langue celtique. Mais ceux-ci renvoient-ils précisément à l'Armorique ? Suffisentils pour affirmer l'origine même du texte copié dans des manuscrits qui montrent des intérêts très variés pour l'Église irlandaise et britannique, mais aussi les lois franques et romaines?

\section{Manuscrit A : Orléans, bibliothèque municipale, 221}

L'écriture de ce manuscrit indique qu'il a été copié au début ou au milieu du IX ${ }^{\mathrm{e}}$ siècle. Il fut ensuite conservé au monastère de

63. L. M. DAvieS, «Isidorian Texts and the Hibernensis», Peritia 11, 1997, p. 207249.

64. Ibid., p. 234.

65. Ibid., p. 235-236.

66. L'édition de Wasserschleben reste insuffisante. 
Fleury. Il se présente de façon générale comme un recueil d'anciens canons ${ }^{67}$, et comporte principalement ${ }^{68}$ :

- les Extraits du livre de David, p. 1-16;

- des extraits et des éléments de comput d'Adam à Stilichon (408), empruntés à Orose, Eusèbe de Césarée, Jean Cassien, Sulpice Sévère, Hermas et le Nouveau Testament, p. 16-21 ;

- des traités sur le dimanche ${ }^{69}$ et la création du monde ${ }^{70}$, qui s'achèvent p. 22 par une formule de copiste «Frères, priez Dieu pour moi, copiste de ce petit livre, afin qu'il remette largement mes dettes en grand nombre ${ }^{71} \gg$.

Son insertion dans la continuité de la copie dans les manuscrits A et $\mathrm{B}$, ainsi que sa présence dans le manuscrit $\mathrm{P}$ laissent à penser, comme le suggère L. Lemoine ${ }^{72}$, que ce colophon, établi pour un manuscrit antérieur, a été inséré dans le corps du texte recopié, contrairement à celui de la p. 212.

- la Collectio canonum hibernensis dans sa version A, p. 23-206;

67. Il est d'ailleurs décrit comme «92. Breviarium collectum decanonibus diversorum conciliorum. - 193» dans le Catalogus librorum qui in bibliotheca coenobii Benedictini Floriacensis ad Ligerim reperti sunt postridie nonas decembris, anno Domini 1552, publié par Léopold DELISLE, Notice sur plusieurs manuscrits de la bibliothèque d'Orléans, Paris, 1883, p. 70-83.

68. Description complète de ce manuscrit dans le Catalogue des manuscrits médiévaux de la bibliothèque municipale d'Orléans, E. Pellegrin et J.-P. Bouhot dir., CNRS éditions, Paris, 2010, p. 304-308 et Hubert MorDEK, Kirchenrecht und Reform im Frankenreich. Die Collectio Vetus Gallica, die älteste systematische Kanonessammlung des fränkischen Gallien, Beiträge zur Geschichte und Quellenkunde des Mittelalters 1, Berlin/New York, 1975, p. 256.

69. Dies dominica I, R. McNally éd., Scriptores Hiberniae minora, Turnhout, Corpus Christianorum, S. L. 108b, 1973, p. 181-182.

70. À partir du livre d'Esdras et non d'Enoch, Voir la préface de R. MCNALly, op. cit., p. 177, contredite par le Catalogue des manuscrits de la bibliothèque municipale d'Orléans, cité note 57, p. 306.

71. Orléans, Bibliothèque municipale 221, p. 22, 1. 29-30 : Pro me frater, oraueris pictore parui codicis deum ut mea debita largiatur in numera. Louis LEMOINE, "Contribution à la reconstitution des scriptoria bretons du haut Moyen Age», Archivum latinitatis medii aevi, 59 (2001), p. 261-268, ici p. 262, ajoute «ac desiderata», mais je n'ai pas vu d'où venait cet ajout. Néanmoins, je n'ai pu consulter que le microfilm en noir et blanc tiré du manuscrit et la fin de la phrase est peut-être visible sur l'original.

72. L. Lemoine, op. cit. à la note précédente. 
- les Extraits des livres des Romains et des Francs, p. 206-212, dont un dernier chapitre copié après les sept premiers canons d'Adamnan ${ }^{73}$, p. 212.

Sur cette dernière page, sur les deux dernières lignes ${ }^{74}$, se trouve inscrit, de la même main que le reste du manuscrit : «Junobrus a écrit ces saints conciles : demande que son âme soit en paix, qu'il demeure et habite (aux cieux ?) sans $f\left(n^{75} \gg\right.$, un colophon qui, cette fois, semble bien faire allusion à la copie de ce manuscrit précis.

L'écriture du manuscrit est diversement qualifiée : «Insular (Breton) minuscule» ${ }^{76}$ pour L. Bieler, elle est décrite comme écriture aux "caractères anglo-saxons» par le Catalogue Général des Manuscrits des bibliothèques publiques de France ${ }^{77}$, tandis que le catalogue édité par E. Pellegrin et J.-P. Bouhot considère qu'il s'agit d'une «écriture bretonne» ${ }^{78}$. Suivant $\mathrm{H}$. McKee, elle ressemble à la main $\mathrm{F}$ qui a glosé le manuscrit Cambridge, University Library, MS Ff.4.42 (1285), dans un milieu combinant influences irlandaises et galloises ${ }^{79}$. L. Fleuriot qualifiait quant à lui l'écriture de «miinsulaire mi-continentale» ${ }^{80}$. Ces variations soulignent la difficulté à préciser l'origine d'une écriture qui combine des éléments issus du monde franc et du monde britannique, ce qui est une impasse souvent rencontrée pour rechercher l'origine de manuscrits de l'époque carolingienne.

En effet, l'écriture anglo-saxonne possédait des caractéristiques

73. Voir l'édition par L. Bieler, 1963, p. 177-181. Sur ces Canons d'Adamnan, voir Allen FrANTZEN, Les Libri paenitentiales, mise à jour du fascicule 27 par Cyrille Vogel, Typologie des sources du Moyen Âge occidental, Turnhout, 1985, p. 21.

74. Il faut corriger sur ce point L. LEMOINE, op. cit. note 61, p. 262 qui donne à tort la p. 211.

75. Orléans, bibliothèque municipale 221, p. 212 : Junobrus scripsit hec sancta sinoda: dicite animam eius in requiem, erit et habitaret in bapo sine fine.

76. BIELER, 1963, p. 12.

77. Catalogue Général des Manuscrits des Bibliothèques publiques de France, consulté le 10/09/2013 : http://ccfr.bnf.fr/portailccfr/jsp/index_view_direct_ anonymous.jsp?record=eadcgm:EADC:D18011856.

78. Catalogue des manuscrits médiévaux de la bibliothèque municipale d'Orléans, op. cit. note 68 , p. $304-308$.

79. H. McKeE, «Scribes and Glosses from Dark Age Wales: The Cambridge Juvencus Manuscript», Cambrian Medieval Celtic Studies 39, summer 2000, p. 1-22, ici p. 10.

80. L. Fleuriot, Dictionnaire des gloses en vieux Breton, Paris, 1964, ms n³ 3, p. 4. 
particulières, au VII ${ }^{\mathrm{e}}$ siècle, bien distinctes de la tradition continentale, mais pour certains traits influencés par la tradition irlandaise ${ }^{81}$. La participation des clercs anglo-saxons aux missions continentales sous autorité franque, en Frise (par exemple Willibrord, † 739) comme en Saxe (Boniface, $\uparrow 754$ ) et à la Renaissance carolingienne (Alcuin, $\uparrow$ 804) fut telle au VIII siècle que des érudits formés à cette écriture anglo-saxonne furent à leur tour les maîtres de multiples clercs actifs au sein du monde franc ${ }^{82}$. Cette influence ne décrut que lentement. Ainsi, pour B. Bischoff, «à partir de 820 environ, Fulda demeure le seul centre d'écriture anglo-saxonne en Allemagne [...] Après l'époque de Raban Maur ( $† 856)$, qui lui-même écrivait une minuscule au caractère anglo-saxon atténué, cette écriture n'a pas tardé à disparaitre de Fulda ${ }^{83}$ ». L'usage de traits insulaires dans un manuscrit copié au début du IX ${ }^{\mathrm{e}}$ siècle peut donc correspondre à de nombreux espaces différents : Angleterre, mais aussi Franconie, Westphalie, Hesse...

B. Bischoff considère que les Armoricains utilisaient une écriture d'origine irlandaise aux VIII ${ }^{\mathrm{e}-\mathrm{IX}^{\mathrm{e}}}$ siècles et qu'ils adoptèrent en général la minuscule caroline au IX ${ }^{\mathrm{e}}$ siècle $^{84}$. En définitive, il reconnait que :

«Parmi les manuscrits bretons écrits au $\mathrm{IX}^{\mathrm{e}}$ et au début du $\mathrm{X}^{\mathrm{e}}$ siècle en minuscule caroline, seul Orléans 221 (193) (un de ses scribes s'appelle Junobrus) présente encore des $g$ insulaires. On distingue souvent l'écriture bretonne soit à cause de ses formes anguleuses soit à cause de ses formes rondes particulièrement évoluées. On peut l'identifier par l'usage des abréviations insulaires et par la présence fréquente de gloses en langue bretonne. Les manuscrits bretons sont également caractérisés par d'assez nombreuses souscriptions en latin hispérique dont les autres monuments nous ont été principalement transmis en Bretagne ${ }^{85}$.»

81. À propos de ces liens du VII siècle, voir Michael Richter, «The English link in Hiberno-Frankish relations in the seventh century», dans Ireland and northern France. A.D. 600-850, Dublin, 1991, p. 95-118.

82. Bernhard Bischoff, Paléographie de l'Antiquité romaine et du Moyen Âge occidental, traduit par Harmat Atsma et Jean Vez, Paris, Picard, 1985, p. 102-107.

83. Ibid., p. 106-107.

84. Ibid., p. 101.

85. Ibid., p. 132. 
On voit ici que le célèbre paléographe est parti de la conviction que les manuscrits glosés en breton étaient des manuscrits bretons et qu'il a tenté, à partir de ces manuscrits, de définir des caractéristiques propres à une écriture bretonne, qu'il n'a à vrai dire pas trouvées puisque son argumentation hésite entre des formes rondes ou anguleuses, puis revient au contenu des manuscrits (gloses bretonnes et hispériques) et expose qu'il s'agit de combinaisons variables de la minuscule caroline et de traits insulaires. Pourtant, la reconnaissance par B. Bischoff de l'origine bretonne de ces manuscrits, bien qu'elle soit fondée, en fait, sur les classifications linguistiques des gloses, a été conservée dans l'historiographie comme si elle reposait sur la paléographie ${ }^{86}$.

Le caractère armoricain de l'écriture ne pouvant être établi indépendamment de la question de la langue des gloses, le lien entre ce manuscrit A conservé à Orléans et la Bretagne continentale ne repose plus que sur deux éléments : les gloses en langue brittonique et le nom du copiste.

J. Loth soutenait que Junobrus était un nom breton, car beaucoup de noms comportant les syllabes Juno, Juna ou Junet se trouvaient dans le cartulaire de Redon ${ }^{87}$. L'interrogation de la base de données comportant le cartulaire ne relève qu'une seule notice, comportant un témoin prénommé Juno ou Luno ${ }^{88}$. Apparaissent par deux fois des individus prénommés Juna et ce ne sont que les syllabes Junet qui se trouvent au début d'un grand nombre d'individus mentionnés par le cartulaire de Redon. Ils ne peuvent donc être reliés de façon plus précise au nom du copiste.

L'argument principal pour relier le manuscrit $\mathrm{A}$ à la Bretagne repose donc sur la langue de ses gloses. Ce manuscrit comporte

86. Par exemple, D. Dumville, English Caroline Script and Monastic History : Studies in Benedictinism, A. D. 950-1030, Woodbridge, 1993, p. 144 note 20, renvoie aux études de Bischoff à propos de la «Breton Caroline misnuscule».

87. J. LоTH, op. cit. supra, p. 104-105.

88. Acte $n^{\circ} 214479$ dans Les espaces de la charte, Cédric GIRAUD, Jean-Baptiste Renault et Benoît-Michel Tock, éds., Nancy : Centre de Médiévistique Jean Schneider ; éds électronique : Orléans : Institut de Recherche et d'Histoire des Textes, 2010. (Telma). En ligne : http://www.cn-telma.fr/chartae-galliae/charte 214479 /. Date de mise à jour : Première version, 10 juin 2010.

Mais Aurélien DE Courson, Cartulaire de l'abbaye de Redon, Paris, 1863, p. 309, note 2, hésite en fait entre les lectures Luno et Juno. 
environ 332 gloses en langue brittonique, glissées entre les lignes ou dans la marge par deux mains différentes. L'une serait le copiste du manuscrit, l'autre, postérieure d'un siècle environ ne concernerait qu'une vingtaine de gloses ${ }^{89}$. J. Loth a défendu le caractère armoricain des gloses en soulignant leur distinction d'avec les éléments gallois et corniques et proposé une datation entre la fin du $\mathrm{X}^{\mathrm{e}}$ siècle et le début du XI ${ }^{\mathrm{e}}$ siècle ${ }^{90}$. Ces gloses ont été reprises comme étant en vieux-breton dans les travaux de L. Fleuriot ${ }^{91}$.

Nous trouvons ici une question plus familière aux celtisants qu'aux historiens. À l'époque de Léon Fleuriot, la chronologie de l'histoire des langues brittoniques reposait sur l'idée d'une différenciation précoce. Les travaux récents portent au contraire à considérer qu'il ne serait possible de distinguer, à partir du $\mathrm{IX}^{\mathrm{e}}$ siècle, que des éléments issus de différents dialectes au sein d'une langue brittonique commune : le vieux-gallois, le vieux-breton, le vieux-cambrien et le vieux-cornique ${ }^{92}$. Mais les aires géographiques de ces dialectes ne pourraient être précisées, tandis que dans une même glose pourraient apparaitre, par exemple, des éléments supposés typiques du vieux breton et du vieux gallois ${ }^{93}$. Contrairement aux associations plus précises retenues par L. Fleuriot, les gloses indiquent bien un milieu érudit utilisant une langue brittonique, mais sans aucune certitude quant à son lien avec la petite Bretagne plutôt que le pays de Galles ou la Cornouailles.

Si le manuscrit d'Orléans a certainement été utilisé dans une communauté utilisant latin et vieux-brittonique, les Extraits des livres des Romains et des Francs ont-ils joué un rôle particulier dans celle-ci ? Le manuscrit A est le seul où des gloses en vieux-brittonique concernent précisément ce texte. Or les Extraits y sont insérés dans un ensemble déjà bien structuré avant cette copie : on retrouve

89. Catalogue des manuscrits médiévaux de la bibliothèque municipale d'Orléans, op. cit. note 68 , p. 308.

90. J. Lотн, «Les gloses bretonnes d'Orléans», Revue celtique V, janvier 1881, p. $104-115$.

91. L. Fleuriot, Dictionnaire des gloses en vieux breton, op. cit., p. 4.

92. Alexandre I Falileyev, Le Vieux-Gallois, Postdam, 2008 (traduction de l'édition russe de 2002), chapitre 1.

93. Ibid., chapitre 4 : «les inclusions du vieux-gallois dans d'autres langues», p. 4051. 
le même enchaînement de textes dans le manuscrit B, et il n'y a pas de rupture dans les rubriques des différentes parties. Leur enchaînement est même parfois confus :

- à la page 23 deux paragraphes de la Collectio canonum hibernensis $^{94}$ sont collés au texte précédent. Vient alors l'invocation Emanuel, un retour à la ligne et une lettre ornée pour annoncer le début du reste de la collection canonique ${ }^{95}$;

- la fin de la Collectio canonum hibernensis est peu claire, puisque deux sauts de ligne, p. 206, séparent le début du chapitre $17 \mathrm{du}$ livre 38 : De doctore bono non contristando ${ }^{96}$ du reste du texte, avant qu'une lettre ornée n'introduise le titre Incipiunt excerpta de libris romanis et francorum, à la ligne 18 ;

- l'enchaînement entre la fin des Extraits des livres des Romains et des Francs, et les Canons d'Adamnan, p. 212, est de nouveau flou, puisque le paragraphe qui annonce distinctement Incipiunt canones Adamnani reprend aussi une partie du texte identifié par L. Bieler comme la disposition A 63 des Extraits.

Pour le copiste Junobrus, l'ensemble des éléments qu'il copie a donc une grande cohérence, au point de mal distinguer l'enchaînement des différents textes rassemblés par son modèle. L'existence de l'ensemble comme un corpus déjà constitué est illustrée par le manuscrit B, qui reproduit exactement le même enchaînement de textes dans sa première partie. Cette considération globale des textes copiés apparaît dans le colophon de Junobrus, où il les qualifie de sancta sinoda, considérant l'ensemble comme des canons conciliaires sans distinguer la collectio canonum hibernensis des autres textes rassemblés. Or, ce n'est que dans ce manuscrit A que le texte même des Extraits des livres des Romains et des Francs a été complété de termes en langue brittonique et c'est dans le cadre d'une glose complète de tous les textes rassemblés au sein du manuscrit.

Les gloses en langue brittonique nous indiquent ainsi l'intérêt global suscité par l'ensemble de cette tradition religieuse, héritée en partie du christianisme insulaire, mais ne prétend pas trier suivant des

94. Orléans, bibliothèque municipale, 221, p. 23, 1. 20-23, qui reprend la collectio canonum hibernensis, I, chapitre 22, b et c, p. 12 dans l'édition de Wasserschleben. 95. Ibid., 1. 24 : De episcopo capitula 22.

96. Ibid., p. 206, 1. 15-17. Édition Wasserschleben, p. 145. 
appartenances ethniques qui ne jouent pas de rôle discriminant dans le corpus. Ainsi, dans la Collectio canonum hibernensis, quelques qualificatifs viennent indiquer le lieu d'un concile, ou distinguer différents individus portant le même nom, comme Gregorius romanus par exemple. Mais les dispositions dont l'origine est ainsi évoquée sont supposées s'appliquer à tout chrétien. Quand deux lois y sont évoquées ${ }^{97}$, c'est uniquement l'Ancien et le Nouveau Testament.

L'ensemble composé par cette collection canonique et les textes brefs qui lui sont rattachés, comme les calculs se référant au consulat de Stilichon, en 400 ap. J.-C., cherche à présenter de façon ordonnée l'héritage de l'ensemble de l'Église d'occident depuis l'Antiquité chrétienne. Les distinctions ethniques n'y jouent pas un rôle structurant. Comme le montre bien l'association des Romains et des Francs dans le titre des Extraits, il s'agit de récupérer tous les héritages pour construire une société chrétienne et c'est cet héritage, dans son ensemble, qui a été glosé pour être compris par un lecteur plus familier avec le vieux-brittonique qu'avec le latin.

\section{Manuscrit B : Paris, BNF, latin 3182 (dit Bigotianus)}

Ce manuscrit composé aux IX $\mathrm{X}^{\mathrm{e}}-\mathrm{X}^{\mathrm{e}}$ siècles a été conservé à l'abbatiale de Fécamp, où il se trouvait sans doute à partir du XII ${ }^{\mathrm{e}}$ siècle ${ }^{98}$. Il est apparenté au précédent, puisqu'il comporte aux pages 1 à 164, sur deux colonnes cette fois, exactement le même enchaînement que l'ensemble des pages 1 à 212 du manuscrit d'Orléans ${ }^{99}$. En revanche, la suite des feuillets contient un enchaînement assez proche du manuscrit $\mathrm{P}$, dont l'unité est faite par ses préoccupations ecclésiastiques.

Dans la logique son projet éditorial, L. Bieler souligne particulièrement les éléments du recueil manuscrit qui lui semblent se rattacher à la tradition irlandaise : les Canons d'Adamnan, présents

97. Collectio canonum hibernensis, livre I, ch. 3, Wasserchleben p. 3.

98. Catalogue des manuscrits de la BNF en ligne, consulté le 11/09/13 :

http://archivesetmanuscrits.bnf.fr/ead.html?id=FRBNFEAD000061028.

Le manuscrit B est consultable en ligne sur le site Gallica.bnf.fr. La page 19 du manuscrit contient l'obit d'un «Godefredus sacerdos» qui a offert le livre à l'établissement de la Sainte-Trinité.

99. Voir le tableau établi par BIELER, 1963, p. 21. 
par trois fois ${ }^{100}$, les canones Hibernenses ${ }^{101}$, des extraits du pénitentiel de Finnian ${ }^{102}$, l'ensemble allant de la préface de Gildas sur la Pénitence, le Synode Aquilonalis Britanniae ${ }^{103}$, et le Synode du bois de la victoire, aux Extraits du livre de David ${ }^{104}$ et au pénitentiel dit Bigotianus $^{105}$.

La première partie, commune avec le manuscrit $\mathrm{A}$, reprend un ensemble déjà constitué, où les différents textes ont fini par empiéter les uns sur les autres ${ }^{106}$ tandis que le manuscrit comporte aussi de nombreux éléments en lien avec l'Église anglo-saxonne : les canons de Théodore ${ }^{107}$, des extraits des pénitentiels attribués à Egbert et à Bède ${ }^{108}$. Trois lettres de Grégoire le Grand encadrent la

100. La première fois ne sont donnés que le début des Canons d'Adamnan, comme dans le manuscrit A, can. 1-7 p. 164, puis p. 173-5 est copié le texte complet, puis p. 283, seulement la fin.

101. Canones Hibernenses (ii-vi chez Wasserschleben) : dans ce manuscrit, p. 177f, 279-280, 302, 305-7, 312 ; édition et traduction BIELER, 1963, p. 160-175. Voir A. FrantZen, op. cit, p. 21.

102. Dans ce manuscrit, p. 176-177 ; édition et traduction BIELER, 1963, p. 74-95. Voir A. Frantzen, op. cit., p. 64.

103. Sur ce texte, les hypothèses de G. LE DUC semblent reposer sur une surinterprétation de la mention tardive et isolée d'Aquilonia, comme le soulignent P. R. Giot, «L'histoire naturelle des hommes d'Armorique et de Bretagne», Les premiers Bretons d'Armorique, P.-R. Giot, Ph. Guigon et B. Merdrignac, Paris, 2003, p. 31-74, ici p. 45 et B. MERDRIGNAC, «La chrétienté bretonne des origines au VIe siècle», ibid., p. 75-89, ici p. 87.

104. Dans ce manuscrit, Praefatio Gildae, Synodus Aquilonalis Brittaniae, Synodus Luci Victoriae, Excerpta de libro Davidis : p. 280-283 ; édition et traduction Bieler, 1963, p. 60-73. Voir A. Frantzen, op. cit., p. 19.

105. Dans ce manuscrit, paenitentiale Bigotianum : p. 286-99; édition et traduction Bieler 1963, p. 198-239. Voir A. Frantzen, op. cit., p. 24.

106. Ainsi, alors que la p. 19 semble avoir été initialement laissé blanche, sauf deux lignes, pour marquer une rupture dans la copie, on retrouve la même imbrication des textes que pour Orléans, bibliothèque municipale, 221, p. 23, 1. 20-23, où sont repris et collés au texte précédent deux éléments de la collectio canonum hibernensis, I, avant le titre qui annonce les chapitres concernant les évêques, au début de la p. 20.

107. Titre p. 164-165 : Incipiunt iudicia Theodori greci et episcope Saxonum. Théodore de Tarse fut archevêque de Canterbury de 668 à 690. Voir Roy FLECHNER, «The Making of the Canons of Theodore», Peritia 17-18 (2003-4), p. 121-143.

108. A. Frantzen, "The penitentials Attributed to Bede», Speculum 58 (1983), p. 573-597 étudie les liens entre ces deux textes, mais ne cite pas ce manuscrit. 
mission d'Augustin de Canterbury pour convertir les Angles : ses réponses à Augustin ${ }^{109}$, une lettre à l'évêque Etherius de Lyon et une lettre à Brunehaut. La diffusion de ces lettres semblerait provenir initialement de l'Église de Lyon, ce qui n'est pas le cas de l'échange du pape avec Félix, évêque de Sicile recopié un peu plus loin ${ }^{110}$.

La continuité avec l'Antiquité chrétienne est soulignée dans ce recueil par les éléments de comput, les nombreux extraits de canons ${ }^{111}$, présentés aussi bien dans une collection en ordre chronologique, la collection dite Dyonisio-Hadriana ${ }^{112}$ qu'en ordre thématique, avec la Collectio canonum hibernensis ${ }^{113}$, qui forme l'essentiel de la base commune avec le manuscrit A ; ainsi que les extraits du Liber Pontificalis jusqu'à Boniface III, pape en 607. La nouvelle organisation de l'Église sous la houlette du souverain franc et du pape apparaît dans le poème adressé par le pape Hadrien à Charlemagne ${ }^{114}$, qui ouvre la collection canonique, des extraits de deux capitulaires de ce souverain publiés en 803 , et deux lettres de Théodulfe. La présence de la loi salique, dans sa version $\mathrm{K}$, c'est-à-dire celle révisée sous l'autorité de Charlemagne, peu avant la fin de ce manuscrit, est assez surprenante, car elle est le seul texte, avec les Extraits des Livres des Romains et des Francs, à ne pas concerner des affaires ecclésias-

Sur les pénitentiels attribués à Théodore, Egbert, archevêque d'York, mort en 766, et Bède, moine en Northumbrie, mort en 735, voir A. FrANTZEN, Les Libri paenitentiales, mise à jour du fascicule 27 par C. Vogel, Typologie des sources du Moyen Âge occidental, Turnhout, 1985, p. 25 à 29.

109. Sur les liens entre les canons de Théodore et le Liber Responsionum, voir Roy FLECHNER, op. cit. supra, p. 135-138.

110. Bruno JUDIC, «La production et la diffusion du registre des lettres de Grégoire le Grand», Les échanges culturels au Moyen Âge. Actes des congrès de la Société des historiens médiévistes de l'enseignement supérieur public, 32e congrès, Dunkerque, 2001, p. 71-87, ici p. 74.

111. Ce manuscrit comporte p. 182-183 des extraits de la concordia canonum de Cresconius. Voir H. MORDEK, Kirchenrecht und Reform im Frankenreich..., op. cit. note 68 , p. $253-255$.

112. Dans ce manuscrit, p. 183-264. Voir H. MordeK, op. cit., p. 151-159 : cette collection donnée par le pape Hadrien Ier à Charlemagne à Rome en 774 a circulé de façon complémentaire avec la collection de canons systématique dite Vetus Gallica, elle aussi diffusée à partir de la cour carolingienne.

113. Ibid., p. 257.

114. E. DümmLER éd., Poetae latini medii aevi I, Berlin, 1881, MGH, p. 90-91. 
tiques ${ }^{115}$. L'enchaînement des capitulaires de 803 et de la loi salique dans sa version carolingienne est néanmoins courant ${ }^{116}$.

L'unité du recueil manuscrit semble reposer sur la construction d'une société chrétienne, sous différentes autorités : les évêques qui formulèrent les canons; les évêques et les docteurs de la foi qui rédigèrent les pénitentiels ou différentes lettres, les papes qui organisèrent des missions. L'autorité du roi franc apparait aussi à travers des capitulaires et la révision de la loi salique. Les divers documents copiés ici illustrent la rencontre des traditions chrétiennes continentales et insulaires qui caractérise l'Église à l'époque carolingienne. Cette sélection n'indique aucun espace particulier dans une Europe occidentale soumise dans son ensemble à l'influence idéologique de la Réforme carolingienne. Celle-ci a intégré la Collectio canonum hibernensis et les pénitentiels insulaires, comme le montre notamment la rédaction du pénitentiel continental, dit Bigotianus. L'attribution de l'origine du manuscrit $\mathrm{B}$ à l'Armorique ne reposait donc que sur deux éléments, comme pour le manuscrit précédent : les gloses en langue brittonique et le nom du copiste.

Trois gloses en vieux breton ont été repérées et éditées par W. Stokes ${ }^{117}$. Les deux premières concernent le texte de la Collectio canonum hibernensis ${ }^{118}$, la troisième l'un des Canones hibernenses ${ }^{119}$. Ces trois gloses en langue brittonique doivent être comparées aux multiples corrections et compléments apportés aux différents textes en latin. La Collectio canonum hibernensis est ainsi très souvent corrigée et complétée ${ }^{120}$ de même que la Collectio

115. Dans ce manuscrit, p. 315-338, on trouve successivement le prologue long de la loi salique, la liste des titres, puis le texte. Dans l'édition de la loi salique par K.A. ECKHARDT, op. cit. note 12, ce manuscrit porte le sigle K 40.

116. H. MoRdeK, Bibliotheca capitularium regum Francorum manuscripta, Munich, 1995, p. 433-435.

117. W. Stokes, «Old breton gloses», Revue celtique, 4, 1880, p. 324-346, ici, p. 328 et p. 345-346. Cette édition a été utilisée par L. Fleuriot, Dictionnaire des gloses en vieux breton où $\mathrm{B}$ constitue le manuscrit 27 , mentionné p. 6 , mais il faut y corriger le nombre de gloses de quatre à trois.

118. p. 126, c.1 : saltrocion; p. 138, c.2 : guohethe.

119. p. 312 , c.1, dans la marge : buorth.

120. p. $39,40,41,43,44,45,47,49,50,53,54,61,68,70,78,87,89,91,92,96$, $100,101,106,115,117,130,133,134,136,138,142,144,149,151,152,155$, 159,160 . 
Dyonisio-Hadriana ${ }^{121}$ et le pénitentiel Bigotianus ${ }^{122}$. Ce type de gloses latines apparait pour les textes brefs qui suivent la Collectio hibernensis ${ }^{123}$, dont deux fois pour les Extraits des livres des Romains et des Francs ${ }^{124}$, ainsi que pour les textes brefs de la suite du recueil manuscrit ${ }^{125}$, puis la loi salique dans sa version carolingienne ${ }^{126}$.

Il y a donc plus de cent dix pages où le texte initial a été amendé en latin, pour des corrections ou des ajouts d'informations. Pour certaines gloses longues, comme p. 47, on peut supposer qu'il s'agit d'une main différente de celle du copiste initial. Les trois gloses en langue brittonique sont donc très minoritaires par rapport à la masse des suppléments apportés au texte latin initial. Elles nous indiquent que le manuscrit eut au moins un lecteur connaissant cette langue, mais toutes les autres interventions n'utilisent que le latin et montrent que l'usage du vieux brittonique était très minoritaire dans la communauté des lecteurs de ce recueil.

Comme dans le manuscrit A, un colophon semble avoir été inséré dans le corps de la copie, et correspondre à une étape précédente de la tradition manuscrite ${ }^{127}$. En revanche, la dernière page de ce manuscrit donne un colophon qui semble bien correspondre à cette copie :

«Moi, l'élève Maeloc, j'ai écrit ceci. Que je puisse ainsi, sage, régner avec le Christ sans fin pour des années et des siècles.

121. Corrections et compléments p. 185, 186, 194, 195, 201, 204, 208, 209, 211, 220, 236, 238, 243, 245, 252, 256, 257, 262.

122. p. 286, 287, 289, 292, 294, 297 et 298.

123. p. 172 et 173 sur les Canons de Théodore, p. 174 sur les Canons d'Adamnan; p. 176, rajouts dans la marge sur les canons du concile d'Ancyre.

124. p. 160 et 161 .

125. p. 266 sur une lettre de Grégoire le Grand, p. 274, 278 et 279 sur la collection sur les dîmes, p. 280 sur le texte de Gildas sur la pénitence, p. 281 sur le Synodus Aquiloniae Britanniae, p. 283 sur le Livre de David, p. 284-285 sur des extraits d'Isidore de Séville, p. 299 sur la lettre de Théodulfe d'Orléans, p. 300, 304, 305, 306 et 307 sur les différentes citations rassemblées, p. 308 sur le Liber Pontificalis, p. 342 sur l'échange épistolaire entre Grégoire le Grand et Félix, p. 348 sur le texte de Théodulfe d'Orléans, et p. 355 sur le pénitentiel d'Egbert.

126. Corrections sur la loi salique, p. 321,; p. 325 ; p. 328, p. 329 et p. 333.

127. p. 18, c. 1, 1. 26-28: Pro me frater oraueris, pictore parui codicis, deum ut mea debita largiatur inumera. Voir note 71 supra. 
Quand viendra le Seigneur, il rendra aux fidèles suivant leurs mérites ${ }^{128}$.»

Le nom du copiste semble le relier à une communauté utilisant une langue brittonique.

Ainsi, les recueils manuscrits A et B présentent des ensembles conçus comme cohérents. L'intégralité du manuscrit A (p. 1 à 212) et le premier tiers du manuscrit B (p. 1 à 164) reprennent une sélection déjà opérée par un copiste, sans doute celui qui écrivit le colophon désormais intégré au texte. Ce premier recueil montre l'intérêt porté à une tradition insulaire, à partir de l'héritage de l'antiquité tardive. L'ensemble éveilla un intérêt dans un milieu lettré utilisant une langue brittonique en sus du latin, pour lequel fut glosée en au moins deux étapes l'intégralité du manuscrit A. Les Extraits des livres des Romains et des Francs ne se distinguent pas dans cet ensemble.

Le premier recueil fut repris par un autre copiste portant un prénom brittonique, mais cette fois intégré à la formation d'un nouveau recueil cohérent, le manuscrit $\mathrm{B}$, installant la collection canonique et les autres éléments initiaux dans un ensemble plus vaste, comprenant d'autres écrits insulaires, une autre collection canonique, des éléments sur les papes, les évêques et la réforme législative de Charlemagne. La cohérence de cette sélection repose sur celle de la tradition ecclésiastique depuis le concile de Nicée jusqu'à la réforme carolingienne et montre que l'ambition de construire et réguler une société chrétienne est conçue de manière universelle, pour l'ensemble de l'Empire de Charlemagne. Si différents ensembles ethniques et politiques ont contribué à la normalisation chrétienne de la société, aucun apport n'est spécifiquement distingué, en dehors de la loi salique et des Romains et des Francs évoqués par le titre des Extraits.

La communauté pour laquelle fut copiée ce recueil comportait des membres connaissant le vieux-brittonique, ainsi que l'indiquent

128. Le microfilm du manuscrit ne permet pas de déchiffrer cette inscription et je m'en remets à la lecture proposée par le catalogue en ligne de la BNF, qu'il faut pourtant corriger sur le fait qu'elle se trouve p. 356 (et non 355) : Discipulus Maeloc conscripsi hunc ego (?). Sic sanus nunc omnibus annis temporibusque. Cum Christo valeam regnare tunc sine fine. Cum veniat Dominus reddensque fidelibus apta. 
trois gloses. Mais son usage était très minoritaire, comme le montrent les annotations latines cent fois plus nombreuses qui parsèment le manuscrit. En outre, aucun élément ne permet de préciser l'origine, continentale ou insulaire, du manuscrit offert au XII ${ }^{\mathrm{e}}$ siècle à l'abbaye de Fécamp.

\section{Le manuscript H : Oxford, Bodleian Library, Hatton 42 (section A)}

D'après le catalogue de la bibliothèque ${ }^{129}$, ce manuscrit comporte trois parties distinctes, $\mathrm{A}, \mathrm{B}$, et $\mathrm{C}$, copiées entre le $\mathrm{IX}^{\mathrm{e}}$ et le $\mathrm{XI}^{\mathrm{e}}$ siècle, reliées ensemble, semble-t-il, dès le XII ${ }^{\mathrm{e}}$ siècle ${ }^{130}$. Seule la partie A, qui comporte les Extraits des livres des Romains et des Francs sera donc retenue ici. Copiée dans la deuxième partie du IX $\mathrm{X}^{\mathrm{e}}$ siècle, elle comporte les folios 8 à 142v car le premier cahier (fol. 1 à 7) a été remplacé par un copiste de Christ Church, Canterbury, vers l'an mil ${ }^{131}$. Immédiatement après, le livre fut entre les mains de Wulfstan, évêque de Worchester et archevêque d'York (1002-1023), dont la main est visible en de nombreuses annotations ${ }^{132}$.

Le copiste change au folio 24 recto ; aux folios $56 \mathrm{v}$ et 79 furent insérés des éléments copiés aux $\mathrm{X}^{\mathrm{e}}-\mathrm{XI}^{\mathrm{e}}$ siècles. On trouve dans ce manuscrit :

- la Collectio canonum hibernensis dans sa version B, introduite par un prologue avec des extraits d'Isidore de Séville (fol. 1-130r);

- les Extraits des livres des Romains et des Francs (fol. 130r-132v);

- les Canons d'Adamnan (fol. 132v-133v);

- des extraits des Institutes de Gaius contenu dans le Bréviaire $d^{\prime}$ Alaric $^{133}$ (fol. 314 v-138r);

129. H. H. E. Craster and N. Denholm-Young, Vol. II, part II (1937), n 4117 , p. 848 et 849, dans Falconer Madan et al. dir., A summary catalogue of western manuscripts in the Bodleian Library at Oxford which have not hitherto been catalogued in the Quarto series Oxford, 1895-1953. Dans le cadre de cette étude, je n'ai pu consulter les manuscrits conservés à Londres et à Oxford, ni leur reproduction.

130. H. MORDEK, Bibliotheca..., op. cit., p. 404-406.

131. D. N. Dumville, «Wulfric Cild», Notes and Queries, n. s. 40, 1, 1993, p. 5-9, ici p. 7.

132. D. Dumville, English Caroline Script..., op. cit. note 86, p. 3 note 12 et p. 49.

133. Fol. $134 \mathrm{v}$ : Incipiunt Gaius Institutionum liber I. Lex Romana Visigothorum ad LXXVI librorum manu scriptorium fidem recognovit, Gustal HAENEL éd., Leipzig, Teubner, 1849, p. 314-337. 
- une table de correspondances (fol. 138r-139r) ; aux 133v et 139v se trouvent des notes sur les poids et mesures et les parties du discours ;

- des fragments des jugements de Théodore ont été ajoutés sur l'espace laissé libre aux fol. 142 r et $v^{134}$.

Le catalogue en ligne ${ }^{135}$ évoque aussi des neumes bretons (fol. 101 verso), ainsi qu'une glose en vieil anglais (fol. 49) ${ }^{136}$, et des gloses latines en termes hispériques, ajoutées d'une main du $\mathrm{X}^{\mathrm{e}}$ siècle (fol. 94, 138 et autres). Au folio $79 \mathrm{r}$ apparait le nom de Wulfric, écrit peut-être au XI ${ }^{\mathrm{e}}$ siècle ; le manuscrit porte aussi des annotations de la main de Wulfstan, évêque de Worchester (1002-1016) et archevêque d'York (1002-1023) ${ }^{137}$.

La remarque qui clôt la dernière page : «Ici se termine, au nom de Dieu, ce petit ouvrage ${ }^{138} »$, montre sa conception comme un ensemble cohérent, où les Extraits et le droit romain complètent la collection canonique par leurs préoccupations laïques.

Comme pour les manuscrits précédents, la localisation de la copie de ce manuscrit en Bretagne repose sur 6 gloses en langue brittonique éditées par W. Stokes ${ }^{139}$ - elles portent sur la Collectio canonum hibernensis - ainsi que sur un nom mentionné par un colophon : «que Matguoret me bénisse ${ }^{140} »$. Le nom de Matguorec indique bien l'usage d'une langue brittonique, mais ne permet pas de préciser le lien entre le personnage portant ce nom et le manuscrit. Ces gloses ont été reprises par L. Fleuriot et l'attribution à la Bretagne a depuis été placée sous l'autorité de B. Bischoff ${ }^{141}$.

134. R. FleChner, «The Making of the Canons of Theodore», Peritia 17-18 (20034), p. 121-143, ici p. 132-133.

135. Bodleian Library, Online Catalogues of Western Manuscripts : http://www. bodley.ox.ac.uk/dept/scwmss/wmss/online/medieval/hatton/hatton.html, consulté le 02/10/2013.

136. Voir D. N. Dumville, «Wulfric Cild», art. cité note 131, p. 6.

137. Ibid.

138. Fol. $139 \mathrm{v}$ : Finit hoc opusculum in Dei nomine.

139. W. StOKeS, «Old breton glosses», Revue celtique IV, 1879, p. 324-348, sur ce manuscrit p. 324 et 341-342.

140. Fol. 22 r : matguorec benedic mihi condit, selon Roy FLECHNER, «Paschasius Radbertus and Bodleian Library Hatton $42 »$, The Bodleian library record, 2004, p. 411-421, ici p. 416.

141. Comme par exemple dans R. FleChneR, op. cit. supra, note 30. 
Pourtant, R. Flechner a identifié une autre note au folio $23 \mathrm{r}$, en marge de la Collectio canonum hibernensis : leuite pascasius, qu'il propose, en comparant avec les rares mentions semblables, de relier à Paschase Radbert, abbé de Corbie de 843/844 à sa mort en 860 , qui aurait pu consulter le manuscrit à Corbie ou à Saint-Riquier ${ }^{142}$. Il relève les autres textes d'origine irlandaise qui furent retravaillés dans ces abbayes ou par Paschase lui-même. En conséquence, il souligne que les termes hispériques, les gloses en langue brittonique et le nom de Matguoret ont pu être copiés dans le manuscrit à partir d'un exemplaire breton, ou bien insérés à Corbie par des copistes bretons.

S'il y eut bien une étape où ce manuscrit suscita de l'intérêt pour une communauté utilisant le vieux-brittonique, sans que nous puissions préciser une origine cornique, galloise ou armoricaine, il ne s'affirma pas particulièrement pour les Extraits des livres des Romains et des Francs. Ce groupe était très proche de milieux francs, car Paschase Radbert, suivant l'hypothèse de R. Flechner, eut l'ouvrage en main très peu de temps après sa copie, et de milieux anglosaxons, qui laissèrent leurs marques dans le manuscrit au moins dès l'an mil. Leur intérêt montre bien que les Extraits des livres des Romains et des Francs n'était pas, à leurs yeux, un texte spécifiquement dédié à un groupe ethnique de langue brittonique, et son association à la Collectio canonum hibernensis comme au droit romain revu sous l'autorité d'Alaric montre la volonté d'appliquer la justice dans une société chrétienne, quelle qu'en soit l'autorité supérieure.

\section{Le manuscrit O : Londres, British Library, Cotton Otho E. XIII}

L. Bieler considérait que ce manuscrit avait été copié au $\mathrm{X}^{\mathrm{e}}$ siècle ${ }^{143}$, tandis que le catalogue en ligne de la British Library précise qu'il fut copié entre le dernier quart du $\mathrm{IX}^{\mathrm{e}}$ et le premier quart du $\mathrm{X}^{\mathrm{e}}$ siècle $^{144}$. À la fin du Moyen Âge, ce manuscrit se trouvait à Saint-Augustin de Cantorbéry. Il a reçu des gloses en vieux

142. Ibid.

143. L. Bieler, 1963, p. 14 et p. 22-24.

144. Searcharchives.bl.uk, consulté le 25/11/2013. 
brittonique ${ }^{145}$, mais pas sur les Extraits des livres des Romains et des Francs $^{146}$.

Sur les folios 2 à 180 (le premier est un ajout d'époque moderne), on trouve, parfois très abîmé par l'incendie de la bibliothèque en 1731:

- une préface et un sommaire (fol. $2 \mathrm{v}-3 \mathrm{v})$;

- les Extraits du livre de David (3v-11v);

- la Collectio canonum hibernensis (11v-128r) dans une recension A augmentée par des extraits de la recension B copiés entre les lignes ${ }^{147}$;

- les canons du synode de Rome, en 721 (131 r-v) ${ }^{148}$, sur les mariages interdits ;

- l'Epistola ad episcopos in Campo hAi (132v-133r) ${ }^{149}$, un document lié à saint Patrick ${ }^{150}$;

- le prologue de la recension A de la Collectio canonum hibernensis (136 r) ;

- les Extraits des livres des Romains et des Francs (139r-141v), mais introduit par le titre : sinod(us?) rom(anorum?ae?) incipi(unt?) pauca colomella;

- les Canons d'Adamnan (141v-143r);

- la légende des Sept Dormants ${ }^{151}$ (146v-150r);

- des compléments de la Collectio canonum hibernensis appartenant à la recension $\mathrm{B}(150 \mathrm{r}$ et $\mathrm{v}$ et $152 \mathrm{r}-179 \mathrm{v})$, au milieu desquels un feuillet est consacré aux canons de Théodore $(151 \mathrm{r}-\mathrm{v})^{152}$.

145. L. Fleuriot, Dictionnaire, p. 6, ms. n 21.

146. D. N. Dumville, «On the dating of the early Breton law codes», Études celtiques XXI, 1984, p. 207-221, ici note 7 p. 208.

147. Appelée C par. D. N. Dumville, «Ireland, Brittany, and England..., op. cit. note 57 , p. $85-95$, ici p. 85.

148. La Patrologie Latine présente deux fois ce texte, édité à partir d'autres manuscrits, Patrologia Latina cursus completus, J.-P. Migne éditeur, Paris, $1839-1864$, t. 67 , c. $342-346$ et t. 77 , c. $1339-1340$.

149. № 364 dans Michael LAPIDGE et Richard SHARPE, A bibliography of celtic-latin literature 400-1200, Dublin, 1985, p. 106.

150. Paul GrosjeAn, «Notes sur les documents anciens concernant Saint Patrice», Analecta Bollandiana, 62, 1944, p. 42-73, sur ce fragment p. 44-46.

151. Peut-être BHL 2316.

152. Sur ce manuscrit, voir R. FLECHNER, «The Making of the Canons of Theodore», Peritia 17-18 (2003-4), p. 121-143, ici p. 133 
L. Bieler a relevé de nombreuses variantes qui permettent de rapprocher le texte des Extraits des livres des Romains et des Francs et des Canons d'Adamnan contenus dans les manuscrits $\mathrm{H}$ et $\mathrm{O}^{153}$. L'unité du manuscrit apparait cette fois constituée autour de la discipline ecclésiastique, avec la question du mariage qui fait l'objet du synode de Rome en $721^{154}$. En dehors des gloses, ce manuscrit ne semble pas particulièrement lié à une communauté ethnique, car ses centres d'intérêt de dépendent pas des regroupements politiques. L'inadéquation du titre est moindre si l'on considère que colomella pourrait être une erreur de lecture ou d'écriture pour columnella, les colonnes d'une page. Quoi qu'il en soit, le titre associe les Extraits des livres des Romains et des Francs aux synodes romains, comme celui de 721 qui a été copié quelques pages plus haut. Le copiste assimile donc ces dispositions légales à des canons.

\section{le manuscrit X : Cambridge, Corpus Christi College 265}

Il s'agit d'un ensemble composé pour une communauté monastique sur 273 folios ${ }^{155}$, en deux volumes mêlant latin et vieil anglais ${ }^{156}$ copié de plusieurs mains différentes, à Worchester au milieu $\mathrm{du} \mathrm{XI}^{\mathrm{e}}$ siècle ${ }^{157}$. La formule de contrition qui ouvre le recueil se place sous l'autorité de Wulfstan, sans doute l'évêque de Worchester qui soutint Guillaume après sa conquête et mourut en 1095.

On trouve dans le premier volume de nombreux textes brefs ainsi assemblés :

- des prières, p. 4-7;

- des lettres d'Alcuin, p. 7-17;

153. BIELER, 1963, p. 23 et 25.

154. Rudolf SCHIEFFER, «Gregorius II, Papst», Lexikon des Mittelalters, Stuttgart, 1977-1999, vol. 4, col. 1666-7.

155. Sur ce manuscrit, voir Montague R. JAMES, A Descriptive Catalogue of the Manuscripts of the Library of Corpus Christi College Cambridge, Cambridge, 1909, p. 14-21, dont la description est précisée par le catalogue en ligne de la bibliothèque de Cambridge, consulté le 27/11/2013, sur https://parker.stanford. edu/parker/catalog/P62F9F7C. Le manuscrit est consultable en ligne en couleur à partir de ce même site.

156. K. SCARFE BeCKetT, «Worchester Sauce : Malchus in Anglo-saxon England», Latin learning and english lore. Studies in Anglo-Saxon Literature for Michael Lapidge, vol. II, Toronto, 2005, p. 212-231, sur ce manuscrit, note 56.

157. D. Dumville, English Caroline Script and Monastic..., op. cit. note 86, p. 52. 
- des extraits d'Isidore de Séville, p. 17-20;

- le premier capitulaire de Gerbald de Liège ${ }^{158}$, p. 20-22 ;

- les Excerptiones attribués à Egbert, p. 22-37 ;

- le pénitentiel d'Egbert, p. 37-50 ;

- des extraits du pénitentiel de Théodore, p. 50-51;

- des extraits sur le mariage et la pénitence, p. 58-69;

- des extraits des pénitentiels d'Halitgaire et Théodore, p. 69-71;

- des extraits des canons du concile d'Aix en 816;

- le Manuel en vieil anglais à l'usage d'un confesseur, p. 72-83;

- des extraits de la collection de capitulaires d'Ansegise, p. 83-91;

- des extraits de la regula canonicorum d'Amalaire, p. 91-94;

- des extraits des pénitentiels dont celui de Cuméan, celui dit pseudo-Romanum et celui du pseudo-Théodore, p. 94-96 ;

- trois canons irlandais, p. 96-98;

- des extraits attribués à Théodore et à Egbert, p. 98-100 ;

- les Extraits des livres des Romains et des Francs, p. 100-104. Si la rubrique, p. 100, les annonce bien, la fin est plus floue, mêlant après la disposition A 61 sur l'interdiction de porter des cheveux more barbarorum, à la façon des barbares, un chapitre De tonsura, extrait d'Egbert, puis sous la rubrique can(ones) roman(orum) le canon 6 du premier synode de Patrick ${ }^{159}$ qui condamne tout clerc qui ne porte pas une tunique et la tonsure romaine ;

- des Exempla Saxonica accastigationis hominum, p. 105-112, glosés en vieil anglais p. 112 ;

- des décrets et lettres des papes issus de la collection de Raoul de Bourges, p. 113-121;

- les capitula de Théodulfe d'Orléans, ainsi qu'une lettre de cet évêque, p. 121-160;

- des lettres pastorales d'Aelfric, p. 160-180;

- une homélie de Wulfstan, p. 180-198;

- des canons tirés de la Collectio Dyonisio-Hadriana, p. 199-208;

- des formules de bénédiction et d'excommunication, avec un début de carte, p. 209-215 ;

158. Pour ce capitulaire, lié aux deux synodes ecclésiastiques de 801-802, voir Julien MAQUET, «Faire justice» dans le diocèse de Liège au Moyen Âge. Essai de droit judiciaire reconstitué, Genève, 2008, p. 89.

159. BIELER, 1963, p. 54-55. 
- les lois du roi Edgar d'Angleterre (959-975), p. 216-231.

Après des feuillets blancs viennent :

- la lettre d'Aelfric aux moines d'Eynsham, p. 237-268;

- des extraits du liber officialis d'Amalaire de Metz, p. 268-298;

- des extraits du Pontifical romano-germanique, p. 298-329;

- les Eclogae de ordine Romano attribués à Amalaire de Metz ;

- Bernold de Constance, Micrologus de ecclesiasiticis observationibus, p. 368-442.

Ce manuscrit témoigne de l'assimilation de l'héritage des évêques carolingiens au sein de l'Église anglo-saxonne du $\mathrm{X}^{\mathrm{e}}$ siècle. À sa propre tradition des pénitentiels et de la référence à Rome se joignent donc les pénitentiels et les instructions liturgiques rédigés dans le monde franc, de même qu'est reprise la législation d'Edgar comme des souverains carolingiens. Les éléments les plus récents, le Pontifical romano-germanique et les écrits de Bernold de Constance, favorable à la réforme grégorienne, sont ajoutés à ce socle plus ancien.

Celui-ci forme un tout, comme le montre l'insertion floue des Extraits des livres des Romains et des Francs, qui se distingue à peine des canons du synode réuni par Patrick, ou des extraits relevés par Egbert : l'unité du passage est fournie par l'obligation de porter la tonsure romaine. Ce manuscrit nous montre que les Extraits des livres des Romains et des Francs ont intéressé un milieu utilisant aussi bien le vieil anglais que le latin, et qu'ils ont été intégrés dans l'héritage normatif chrétien de l'époque carolingienne, sans aucune distinction suivant la langue ou l'appartenance ethnique.

\section{Le manuscrit $P$ : Paris, BNF, latin 12021}

Ce manuscrit est composite ${ }^{160}$ : les quatre premiers cahiers de huit pages ont une numérotation de I à IV qui apparait au dos du dernier feuillet. Cette numérotation est indépendante pour la deuxième partie, où l'on trouve les numéros des cahiers, de I à XII ${ }^{161}$. À partir

160. Le microfilm noir et blanc de ce manuscrit est consultable en ligne, sur le site Gallica.bnf.fr.

161. Les cahiers sont numérotés en f. 47 verso : I, f. 55 verso II, f. 63 verso III, f. 71 verso IIII, f. 79 verso V,f. 87 verso VI, f.95 verso VII, f. 103, verso VIII, f. 111 
du folio 33 commence un nouveau système, bicolore, pour les titres et les lettres ornées. En outre, l'ancienne pagination, avant la réunion des deux parties, apparait encore et montre que leur assemblage fut assez tardif. Nous ne retiendrons ici que la deuxième partie, qui commence au folio désormais numéroté $33^{162}$.

Copié au $\mathrm{X}^{\mathrm{e}}$ siècle, ce manuscrit fut conservé à Corbie puis à Saint-Germain-des-Prés. Le recueil suit le même enchaînement que la deuxième partie du manuscrit $\mathrm{B}^{163}$, sauf en ce qui concerne les Extraits des livres des Romains et des Francs qui apparaissent ici dans une recension spécifique, dénommée P par L. Bieler.

L'ouvrage commence par la Collectio canonum hibernensis, dans sa version A, la plus courte. La table des chapitres (fol. 3339), est suivie par un prologue (fol. $39 \mathrm{v}$ ), puis la collection du folio 40 à $127 \mathrm{r}$. Elle se clôt en mentionnant «Ruben de Dairinis et $\mathrm{Cu}$ Cuimni d'Iona ${ }^{164} \gg$. Comme dans la plupart des manuscrits précédents, c'est cette partie qui a fait l'objet du plus de relectures, gloses et annotations, aussi bien en latin qu'en brittonique. En effet, les 11 gloses éditées par W. Stokes ${ }^{165}$ sont consacrées à ce texte ${ }^{166}$. Elles restent néanmoins minoritaires, sur ces pages, par rapport aux multiples annotations en latin portées sur le texte. En dehors de la Collectio canonum hibernensis, le texte latin est souvent corrigé et comporte des marques, mais pas de gloses aussi longues.

verso : VIIII a été coupé mais se devine encore, f. 119 verso X, f. 127 verso : XI, f. 135 verso XII, f. 139 verso : XII aussi.

162. Je ne retiens donc pas exactement le même découpage que celui proposé par H. GUILLOTEL, "Recherches sur l'activité des scriptoria bretons au IXe siècle», Mémoires de la société d'histoire et d'archéologie de Bretagne, tome LXII, 1985, p. 9-36, qui proposait d'étudier comme cohérente la partie allant du folio 40 au folio 139. En revanche, il y a bien une rupture entre le fol. 39 et le fol. 40 : l'écriture passe de 42 à 28 lignes par page et Delphine Mercuzot m'a confirmé par courrier électronique le 5/12/2013 qu'il manquait un feuillet à cet endroit dans ce cahier.

163. BIELER, 1963, p. 24.

164. Voir supra note 57.

165. W. Stokes, Revue celtique IV, 1879, p. 327-328, reprise par L. Fleuriot, Dictionnaire, p. 5 , ms. $\mathrm{n}^{\circ} 11$.

166. 2 gloses fol. 44 r , 2 gloses fol. $45 \mathrm{v}, 1$ glose fol. $46 \mathrm{v}, 1$ glose p. 49 v, 3 gloses fol. $63 \mathrm{r}, 1$ glose fol. $94 \mathrm{v}, 1$ glose fol. $97 \mathrm{r}$. 
Après le folio $127 \mathrm{v}$, on trouve :

- De ordinatione episcopi, f. 127- 132 verso. Il s'agit des jugements de Théodore ${ }^{167}$;

- les Canons d'Adamnan, fol. 132v-133v;

- les canons des conciles d'Ancyre et de Néocésarée, fol. 133v-134r;

- un extrait du pénitentiel de Finnian, fol. 134 r-135r;

- les Extraits des livres des Romains et des Francs, version P, 135r-138r, introduits fol. 135r par le titre : Incip(iunt) iudi(cium) culpar(um), «Ici commencent les jugements des peines»;

- les Canones Hibernenses I et II, fol. 138r-139r. C'est dans ces canons que se trouvent insérés les trois termes ou locutions que W. Stokes considérait comme étant du vieil irlandais ${ }^{168}$. S'il s'agissait antérieurement de gloses, elles sont ici incluses dans le texte latin ${ }^{169}$;

- au deuxième paragraphe du folio $139 \mathrm{r}$ vient un ensemble édité et analysé par L. Lemoine comme une somme de colophons ${ }^{170}$, reprenant une partie de ceux repris dans A et B. Une nouvelle partie, (e), évoque :

«Le clerc Arbedoc a lui-même copié de son écriture sur cinquante-cinq cahiers de cinq feuilles ${ }^{171}$, sur ordre de l'abbé Hael Hucar, ces collections qui ont été assemblées ici dans ce livre à partir des Saintes Écritures ou des sources sacrées, ou bien à partir des canons d'un saint concile que les pères ont établi chez différents peuples et en différentes langues ${ }^{172}$.»

167. BIELER, 1963, p. 22.

168. W. Stokes, Revue celtique, IV, 1879, p. 325, note 1 . Ces passages se trouvent au fol. 138r (Canones Hibernenses I, 4), au fol. 138 verso (Canones Hibernenses II, 5) et 139 recto (canones hibernenses II, 10).

169. Pierre-Yves LAMBERT, «Rencontres culturelles entre Irlandais et Bretons aux $\mathrm{IX}^{\mathrm{e}}-\mathrm{X}^{\mathrm{e}}$ siècles : le témoignage des gloses», Irlande et Bretagne, XX siècles d'histoire, C. Laurent et H. Davis dir., Rennes, 1994, p. 97-106, ici p. 98.

170. Louis LEMOINE, «Contribution à la reconstitution des scriptoria bretons du haut Moyen Âge», Archivum latinitatis medii aevi, 59 (2001), p. 261-268, ici p. 262263. Il faut néanmoins corriger la localisation du passage, qui se trouve en 139 recto et non verso.

171. Si l'on suit l'interprétation de lacinione comme $L V$ ciniones que propose P.-Y. LAMBERT, op. cit. p. 104, note 3 et que défend L. LEMOINE, op. cit. supra. Le latin doit être corrigé plusieurs fois pour pouvoir proposer une traduction. 172. Arbedoc clericus ipse has collectiones conscripsit lacinione se concriptionis 
La copie dans un ensemble de colophons empruntés montre que ce passage ne semble pas correspondre à cette copie manuscrite, mais à une étape antérieure, d'autant que le verso de la page est copié de la même main, semble-t-il, et reprend cette fois les éléments de comput d'Adam à Stilichon qui sont présents dans le manuscrit $\mathrm{A}$. Les noms d'Arbedoc et de l'abbé Hael Hucar semblent ainsi correspondre à des communautés utilisant le brittonique, mais leur lien avec la tradition manuscrite des Extraits des livres des Romains et des Francs ne peut être précisé : le colophon provient-il d'un manuscrit qui les contenait ? En tout cas, la copie de ce colophon ne paraissait pas inadaptée pour le copiste du manuscrit $\mathrm{P}$, qui incorporait donc les Extraits à un ensemble désigné comme des extraits de la Bible et de canons conciliaires.

\section{Conclusion}

L'analyse approfondie des manuscrits a montré la disparition d'une bonne partie des arguments avancés par Léon Fleuriot, puis acceptés par D. N. Dumville ${ }^{173}$ : dès lors que les gloses ne peuvent être attribuées à une communauté linguistique spatialement définie, l'origine des manuscrits, comme du texte des Extraits des livres des Romaons et des Francs devient insaisissable. Rappelons les arguments autrefois avancés :

«L'origine armoricaine du texte résulte : 1) de la tradition manuscrite ; 2) des gloses en vieux breton, à l'exclusion de toute autre langue non latine ; 3 ) des noms des scribes et des abbés mentionnés dans les manuscrits eux-mêmes ; 4) de la comparaison du texte avec les autres lois celtiques, plus particulièrement les lois galloises ${ }^{174}$.»

1) La tradition manuscrite révèle combien les Extraits des livres des Romains et des Francs sont liés à une branche spécifique de la

Hael Hucar abbate dispensente quas sanctis scripturis uel ex diuinis fontibus hic in hoc codice glomerati sunt siue etiam de decreta sanctii patres sinodi qui in diuersis gentibus uel linguis construxerunt.

173. D. N. Dumville, «On the dating of the early Breton lawcodes», Études celtiques XXI, 1984, p. 207-221, accepte de façon générale les arguments de Fleuriot p. 207-210 et ne réfute que la datation proposée.

174. L. FleUriot, 1971, p. 612. 
tradition manuscrite de la Collectio canonum hibernensis (présente dans $\mathrm{A}, \mathrm{P}, \mathrm{B}, \mathrm{H}$ et $\mathrm{O}$ ). Cet ensemble, comme de nombreux textes transmis avec les Extraits (tels les Canons d'Adamnan présents dans $\mathrm{A}, \mathrm{H}, \mathrm{O}$ et $\mathrm{P}$ ) semblent originaires des milieux britanniques liés au christianisme irlandais. Mais comme tous les manuscrits sont postérieurs à 800 , ils reflètent une époque où ces écrits, et notamment cette collection canonique, étaient diffusés dans l'ensemble du monde franc. Il est donc impossible de préciser l'origine insulaire ou continentale des manuscrits à l'aide de ce contenu.

Nous avons vu que les qualificatifs géographiques donnés à leurs écritures ne faisaient que refléter les déductions faites à partir de la langue des gloses, et ne permettaient pas davantage de précision sur leur origine. Quand certains lieux de copie ou de conservation des manuscrits sont repérables, ils indiquent le monde franc (Fleury pour le manuscrit $\mathrm{A}$, peut-être Corbie au IX siècle pour le manuscrit $\mathrm{H}$ ), anglo-saxon (Canterbury vers l'an mil pour le manuscrit $\mathrm{H}$, Worcester au milieu du $\mathrm{XI}^{\mathrm{e}}$ siècle pour le manuscrit $\mathrm{X}$ ), et anglonormand (Fécamp au XII ${ }^{\mathrm{e}}$ siècle pour le manuscrit B, Canterbury à la fin du Moyen Âge pour le manuscrit O). L'ensemble indique bien l'ouest de l'Europe, mais peut tirer son origine aussi bien de la Grande-Bretagne que du continent, tout comme l'utilisation du vieux-brittonique.

2) A, B, H, O et $\mathrm{P}$ ont reçu des gloses en vieux-brittonique. Mais $\mathrm{H}$ et $\mathrm{X}$ comportent des gloses ou des textes en vieil anglais et $\mathrm{P}$ en vieil irlandais. Les Extraits des livres des Romains et des Francs ne reçurent des gloses en vieux brittonique que dans le manuscrit A, dont tout le contenu fut ainsi systématiquement glosé. Néanmoins, dans B comme $\mathrm{P}$, ces gloses vernaculaires sont minoritaires par rapport aux ajouts, corrections et commentaires en latin ajoutés à tous les autres textes, et particulièrement la Collectio canonum hibernensis.

Ces gloses nous indiquent la circulation des manuscrits A, B, H, $\mathrm{O}$ et $\mathrm{P}$ dans des milieux savants connaissant le brittonique et le latin. Ceux-ci pouvaient être situés au Pays de Galles, en Cornouailles ou en Armorique, aussi bien que dans toutes les communautés ayant accueilli des moines et des clercs de ces régions, présentes dans l'ensemble de l'empire franc. 
3) Ces gloses comme certains noms associés aux manuscrits (les copistes Junobrus et Maeloc pour A et B, l'évocation d'un Matguoret pour $\mathrm{H}$, d'Arbedoc sous l'abbé Hael Hucar pour P) indiquent de nouveau des communautés utilisant le brittonique en complément du latin. S'il parait clair que les Extraits des livres des Romains et des Francs y étaient connus et recopiés, cet intérêt n'était nullement exclusif, comme le montrent les noms de Pascasius et de Wulfric copiés sur $\mathrm{H}$ et l'intérêt des évêques anglo-saxons Wulfstan de Worchester, associés à $\mathrm{H}$ et $\mathrm{X}$.

4) Les points communs avec la loi salique, la copie de celleci dans sa version carolingienne dans B, la présence de capitulaires carolingiens dans $\mathrm{B}$ et $\mathrm{X}$, ainsi que du Bréviaire d'Alaric dans $\mathrm{H}$ et des lois du roi Edgar dans $\mathrm{X}$ soulignent à quel point une division suivant des traditions ethniques supposées spécifiques est ici inadaptée. À l'époque carolingienne, toutes les traditions normatives étaient ainsi sollicitées pour l'édification d'une société chrétienne et pouvaient être recopiées très loin de leur lieu d'élaboration initial.

Pour donner un exemple contemporain, en 821, l'évêque Baturic de Ratisbonne demanda la composition d'un important dossier liturgique et canonique contre l'adoptianisme, autour du dossier épistolaire lié au concile de Francfort en 794. Le copiste chargé de ce travail choisit d'y insérer aussi bien des extraits de la Collectio canonum hibernensis, du synode attribué à saint Patrick, que du pénitentiel attribué à Théodore de Canterbury et des capitulaires de Charlemagne ${ }^{175}$. Dans la perspective de la réforme chrétienne de la société, peu importait le lieu où s'étaient tenus les conciles ou les auteurs des recueils, dès lors qu'ils s'accordaient à la tradition de l'Antiquité chrétienne pour christianiser les jugements et les comportements et qu'ils pouvaient être compris à l'aide du latin.

Cette négligence de la source précise du texte apparait bien dans la tradition manuscrite des Extraits des livres des Romains et des Francs : anonymes, ils sont insérés dans un ensemble principalement

175. Il s'agit du manuscrit conserve à Munich, Bayerische Staatbibliothek, latin 14468. Ce manuscrit est mentionné par Florence Close, Uniformiser la foi pour unifier l'Empire. La pensée politico-théologique de Charlemagne, Bruxelles, 2011, p. 103 et décrit par H. MORDEK, Bibliotheca capitularium regum Francorum manuscripta, Munich, 1995, p. 335-339. 
composé de canons et de pénitentiels, se plaçant sous des autorités diverses, insulaires comme continentales. Au sein de ces recueils, ils ne se distinguaient pas, au point que des nombreux empiètements eurent lieu avec les textes voisins.

Dans cette perspective, que les Extraits des livres des Romains et des Francs aient été insérés au résumé latin des lois galloises ${ }^{176}$ montre simplement la continuité de ce point de vue : ces règlements ont paru anciens, et mériter une telle inclusion. Cela n'indique nullement qu'ils aient été spécifiques à un groupe ethnique, ce que contredit directement leur titre.

À l'issue de cette étude, il n'y a donc plus lieu de parler de «Lois anciennes des Bretons d'Armorique», car le lien de la tradition manuscrite avec des communautés utilisant le vieux brittonique ne permet pas de trancher quant à l'origine de ces dispositions légales, entre les îles ou le continent. Les Extraits des livres des Romains et des Francs constituent un texte normatif, principalement recopié dans des communautés utilisant le vieux-brittonique, dont la localisation ne peut être davantage précisée. Il ne reprenait apparemment pas des traditions ethniques spécifiques à ces communautés ni n'était consulté exclusivement pout des jugements en leur sein. Il témoigne au contraire de leur insertion dans les réseaux de savoir européens et de leur participation à l'effort partagé de constitution d'une société chrétienne, qui souhaitait s'appuyer sur le meilleur de toutes les traditions antérieures.

176. D.N. Dumville, «On the dating of the early Breton lawcodes», Études celtiques XXI, 1984, p. 207-221, ici p. 208. 